\title{
Stability and Drift of Underwater Vehicle Dynamics: Mechanical Systems with Rigid Motion Symmetry
}

\author{
Naomi Ehrich Leonard * \\ Department of Mechanical and Aerospace Engineering \\ Princeton University \\ Princeton, NJ 08544
}

\author{
Jerrold E. Marsden ${ }^{\dagger}$ \\ Control and Dynamical Systems \\ California Institute of Technology 107-81 \\ Pasadena, CA 91125
}

July, 1995; this version: March 23, 1997

Physica D 105, (1997), 130-162.

\begin{abstract}
This paper develops the stability theory of relative equilibria for mechanical systems with symmetry. It is especially concerned with systems that have a noncompact symmetry group, such as the group of Euclidean motions, and with relative equilibria for such symmetry groups. For these systems with rigid motion symmetry, one gets stability but possibly with drift in certain rotational as well as translational directions. Motivated by questions on stability of underwater vehicle dynamics, it is of particular interest that, in some cases, we can allow the relative equilibria to have nongeneric values of their momentum. The results are proved by combining theorems of Patrick with the technique of reduction by stages.

This theory is then applied to underwater vehicle dynamics. The stability of specific relative equilibria for the underwater vehicle is studied. For example, we find conditions for Liapunov stability of the steadily rising and possibly spinning, bottom-heavy vehicle, which corresponds to a relative equilibrium with nongeneric momentum. The results of this paper should prove useful for the control of underwater vehicles.
\end{abstract}

\section{Contents}

1 Introduction $\quad \mathbf{2}$

1.1 Summary and Background . . . . . . . . . . . . . . . . 2

1.2 The Nature of the Stability Results Obtained . . . . . . . . . . . . . . . . 3

1.3 Summary of Results for the Underwater Vehicle . . . . . . . . . . . . . . 4

2 Stability Theory for Compact Groups $\quad 6$

2.1 The Basic Setup . . . . . . . . . . . . . . . . . . . 6

2.2 A result of Patrick . . . . . . . . . . . . . . . . . . . 7

2.3 Relation to the Energy-Casimir Method . . . . . . . . . . . . . 8

\footnotetext{
*naomi@princeton.edu. Research supported in part by the National Science Foundation under Grant BES-9502477 and by the Office of Naval Research under Grant N00014-96-1-005.

${ }^{\dagger}$ marsden@cds.caltech.edu. Research partially supported by the National Science Foundation under Grant DMS9302992 and AFOSR Contract F49620-95-1-0419.
} 
3 Stability Theory for Semidirect Products 10

3.1 Reduction by stages . . . . . . . . . . . . . . . . . . 10

3.2 Semidirect Product Stability-Generic Momenta . . . . . . . . . . . . . . . . . 13

3.3 A Double Semidirect Product Stability Theorem . . . . . . . . . . . . . . . . . 14

3.4 Semidirect Product Stability-Nongeneric Momenta . . . . . . . . . . . . . . . 15

4 Dynamics of an Underwater Vehicle $\quad 18$

4.1 Review of the Lie-Poisson form of the Dynamics . . . . . . . . . . . . 18

4.2 Dynamics on the Physical Phase Space . . . . . . . . . . . . . . . . 20

4.3 Stability of Generic Momentum Values . . . . . . . . . . . . . . . . . . 21

4.3.1 Generic Equilibria for a Vehicle with Coincident Centers . . . . . . . . . . . . 21

4.3.2 Generic Equilibria for a Vehicle with Noncoincident Centers . . . . . . . . . . 23

4.4 Stability of Nongeneric Momentum Values . . . . . . . . . . . . . . . . 25

4.4.1 Nongeneric Equilibria for a Vehicle with Coincident Centers . . . . . . . . . . . 25

4.4.2 Nongeneric Equilibria for a Vehicle with Noncoincident Centers . . . . . . . . 27

$\begin{array}{ll}\text { Future Directions } & 31\end{array}$

$\begin{array}{ll}\text { Conclusions } & 32\end{array}$

Appendix: Reduction by Stages $\quad 33$

$\begin{array}{ll}\text { Acknowledgments } & 36\end{array}$

$\begin{array}{ll}\text { References } & 36\end{array}$

\section{Introduction}

\subsection{Summary and Background}

This paper begins with the development of stability theorems for relative equilibria of mechanical systems that have noncompact symmetry groups, such as the group of Euclidean motions of the plane or space. Some of our results apply to certain cases of nongeneric values of the momentum for a mechanical system with symmetry. The techniques used build on the work of Patrick [1992], where a fairly complete picture for the case of compact symmetry groups is given. However, in the noncompact case, the theory for nongeneric equilibria is not as complete.

Our results apply to, and are motivated by, relative equilibria for the dynamics of an underwater vehicle studied by Leonard [1995b] and, in particular, clarify the nature of the stability one obtains. We also study relative equilibria with nongeneric values of their momentum such as a steadily rising or falling, bottom-heavy underwater vehicle. It is because of the coincidence of the direction of gravity and the direction of translation that the value of the momentum for this relative equilibrium is nongeneric.

The results of Patrick [1992] need to be extended for the underwater vehicle example, in both the case of generic and nongeneric values of the momentum, because the properness hypothesis on the action of the symmetry group fails due to noncompactness of this group. In fact, numerical simulations support our claim that there is an additional drift instability in these noncompact 
directions, even for generic values of the momentum, and so this extension is essential; see figure 4.3.2.

We emphasize that while the techniques developed in this paper make use of a substantial amount of theory, the application of these techniques is both straightforward and rewarding as evidenced by our underwater vehicle examples. Further, as explained in Leonard [1995b] and at the end of the present paper, we believe that the general context described here will provide a setting for other interesting developments, such as the study of bifurcations that occur in the dynamics as parameters are varied, the development of control strategies for stabilization and tracking, the study of the effect of dissipation, etc.

Of course, there have been many works on the dynamics of rigid bodies in fluids, and we make no attempt to survey them here. For example, Aref and Jones [1993] study some nonintegrable cases for the dynamics of ellipsoids. Another important paper that treats some topics relevant to the problem, including some interesting comments on the possibility of drift as a source of instability, is Novikov and Shmel'tser [1982]. What makes our paper unique is the inclusion of torques due to gravity and buoyancy, the study of the stability of relative equilibria, including careful attention to attitude and translation drifts and, in related works, the study of the control and stabilization problem.

\subsection{The Nature of the Stability Results Obtained}

The sense in which one has stability requires explanation. It may help to recall that in the study of solitary water waves, one can hope to obtain stability modulo translations at best, since nearby waves will move with slightly different velocities and hence will drift apart from the given wave. A similar thing can happen with an underwater vehicle, but now one must combine possible translational drifts with rotational drifts. Interestingly, the rotational drifts are not arbitrary and can only happen around an axis that stays close to the original axis of spin of the relative equilibrium.

The technique used here is the energy-momentum method developed by Simo, Lewis and Marsden [1991] (see Marsden [1992] for an exposition and further references) combined with the Arnol'd, or energy-Casimir, method (see Holm, Marsden, Ratiu and Weinstein [1985] and Marsden and Ratiu [1994] for summaries and references). These methods have proven to be useful for the study of relative equilibria in mechanical systems with symmetry in a wide variety of applications.

The context for the energy-momentum method is a phase space $P$ with a symmetry group $G$, a $G$-invariant Hamiltonian $H$ and an associated conserved momentum $\mathbf{J}$. The method is used to determine the stability of a relative equilibrium; that is, a point $z_{e} \in P$ whose dynamical orbit is coincident with a one-parameter group orbit. Relative equilibria in many examples including the ones in this paper may be viewed, in the language of Routh (from around 1860) as steady motions.

Patrick [1992] extended the energy-momentum method to the case where the relative equilibrium $z_{e}$ is a regular point for the momentum map (i.e., the derivative of $\mathbf{J}$ is surjective at $z_{e}$ ) but the corresponding value of the momentum $\mu_{e}$ may be nongeneric (this is defined precisely below). A crucial hypothesis in this result is a compactness condition on the isotropy subgroup $G_{\mu_{e}}$, the subgroup of elements of $G$ that fix $\mu_{e}$. His result then gives stability modulo $G_{\mu_{e}}$.

In this paper, we further generalize this result to allow some noncompactness, but at the expense of taking stability modulo a larger group. Our technique is to use theorems on reduction by stages for groups that are semidirect products, which enable one to apply the theorem of Patrick to the stage involving compactness. We also relate the result to the energy-Casimir method. 


\subsection{Summary of Results for the Underwater Vehicle}

We now briefly summarize the results that are obtained for the example of an underwater vehicle. On the theoretical side, we extend the theory to cover the stability analysis for the equilibria studied in Leonard [1995b]. The theory we provide allows us to demonstrate stability modulo the appropriate group, even in the presence of noncompactness, as mentioned above.

The underwater vehicle is modeled as a rigid body moving in ideal potential flow according to Kirchhoff's equations. The vehicle is assumed to be neutrally buoyant (often ellipsoidal), but not necessarily with coincident centers of gravity and buoyancy. We fix an orthonormal coordinate frame to the body with origin located at the center of buoyancy and axes aligned with the principal axes of the displaced fluid as shown in Figure 1.3.1. When these axes are also the principal axes of the body and the vehicle is ellipsoidal, we let the inertia matrix of the body-fluid system be denoted by $I=\operatorname{diag}\left(I_{1}, I_{2}, I_{3}\right)$ and the mass matrix by $M=\operatorname{diag}\left(m_{1}, m_{2}, m_{3}\right)$; note that these matrices include the "added" inertias and masses due to the fluid. The mass of the body alone is denoted $m$ and the acceleration of gravity is $g$. The vector from the center of buoyancy to the center of gravity with respect to the body-fixed frame is $l e_{3}$, where $e_{3}=(0,0,1)^{T}$ indicates that the two centers are assumed to be aligned along the third principal axis. When the body is oriented so that the body-fixed frame is aligned with the inertial frame, the third principal axis aligns with the direction of gravity. Further, since our convention is to let the third axis of the inertial frame point "down" as in Figure 1.3.1, the scalar $l$ is positive if the center of gravity is below the center of buoyancy and negative if it is above.

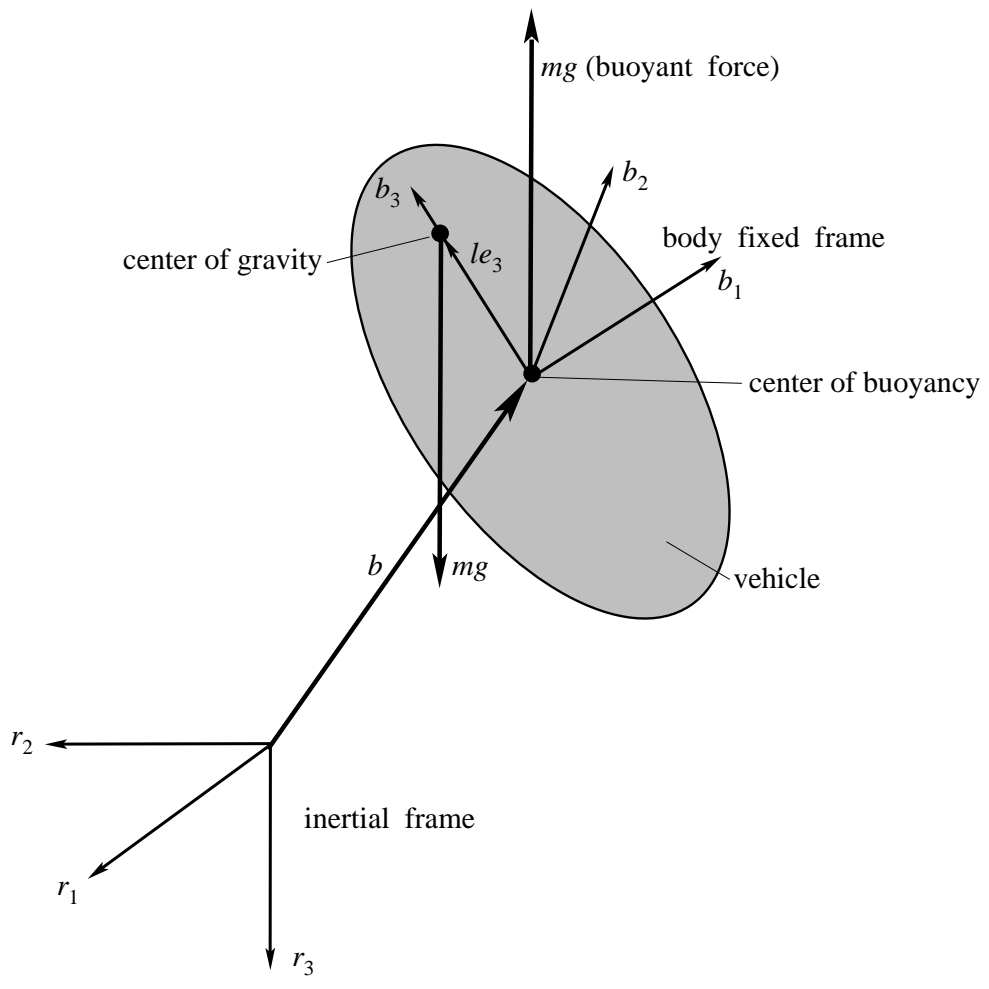

Figure 1.3.1: Schematic of a neutrally buoyant ellipsoidal underwater vehicle. 
1. The first case we study in $\S 4.3 .1$ is that of a vehicle with coincident centers of buoyancy and gravity that translates with momentum (more properly impulse) $P_{3}^{0}$ along one of the principal axes, say the third, and rotates with angular momentum (impulse) $\Pi_{3}^{0}$ about the same axis. In this case, we also suppose that the body is symmetric about the third axis. We show that one has stability of this motion modulo rotations about the third axis and translations in any direction provided that

$$
\left(\frac{\Pi_{3}^{0}}{P_{3}^{0}}\right)^{2}>4 I_{2}\left(\frac{1}{m_{3}}-\frac{1}{m_{2}}\right) .
$$

The physical interpretation of this condition is discussed in $\S 4.3 .1$.

2. The second case we study in $\S 4.3 .2$ is that of a vehicle with noncoincident centers of buoyancy and gravity oriented with the third principal axis parallel to the direction of gravity and translating (but not spinning) with momentum $P_{2}^{0}$ along one of the other principal axes, say the second. We show that one has stability of this motion modulo translations in any direction provided that the system is bottom heavy $(l>0)$ and

$$
m_{2}>m_{1}, \quad m g l>\left(\frac{1}{m_{2}}-\frac{1}{m_{3}}\right)\left(P_{2}^{0}\right)^{2} .
$$

The physical interpretation of this condition is also discussed in $§ 4.3 .2$.

3. The third and fourth cases have nongeneric values of the momentum; that is, as will be evident, they are degenerate instances of the first two cases respectively. The third case is the same as case 1 except that there is no translation; i.e., $P_{3}^{0}=0$. We show that one has stability of this motion modulo rotations about the third axis and translations in any direction provided that the axis of rotation is an axis of symmetry. When symmetry is broken, the rotation is unstable if $I_{3}$ is the intermediate moment of inertia. Unlike the free rigid body, this does not necessarily mean instability of rotation about the intermediate axis of the body; see Leonard [1995b] and $\S 4.4 .1$ for further discussion.

4. The fourth case we study in $\S 4.4 .2$ is that of a rising or falling vehicle with noncoincident centers of buoyancy and gravity oriented with the third principal axis parallel to the direction of gravity; as in the first case, we assume that the body is symmetric about the third axis. Suppose the body translates with momentum $P_{3}^{0}$ along the third axis and rotates with angular momentum $\Pi_{3}^{0}$ about that axis. This case is nongeneric because the direction of translation is parallel to the direction of gravity. We show that one has stability of this motion modulo rotations about the third axis and translations in any direction provided that

$$
m g l>\left(\frac{1}{m_{3}}-\frac{1}{m_{2}}\right)\left(P_{3}^{0}\right)^{2}-\frac{1}{4}\left(\frac{m_{1}}{m_{1} I_{2}-m^{2} l^{2}}\right)\left(\Pi_{3}^{0}\right)^{2} .
$$

5. In the fourth case, some interesting bifurcation phenomena are identified, such as a Hamiltonian Hopf bifurcation (the splitting of eigenvalues off the imaginary axis) and the passing (resonance) of eigenvalues. The detailed investigation of these phenomena is not undertaken here. 


\section{Stability Theory for Compact Groups}

A standard basic strategy for dealing with a system whose symmetry group is the semidirect product of a compact group and a vector space, such as the group of Euclidean motions is to deal with them in two stages, the vector space of translation type variables followed by the compact group of rotation type variables. Therefore, it is appropriate to start in this section with some tools for treating stability in the compact case.

The structure of the section is as follows. We begin in $\S 2.1$ by describing the notation and the setup that will be useful throughout the paper. In $\S 2.2$ we recall Theorem 6 of Patrick [1992] and then in $\S 2.3$ we relate it to the context of systems on Lie groups and the energy-Casimir method. In the case of nongeneric orbits we will need to make use of subcasimirs (defined below) to determine stability. The energy-Casimir context is convenient for doing the calculations. Linking Patrick's result with the energy-Casimir method and making use of subcasimirs is hinted at in the literature (see, e.g., Weinstein [1984]), but is made explicit here.

\subsection{The Basic Setup}

We begin by describing the basic notation and setup that will be useful throughout the theoretical part of the paper. We refer to Marsden and Ratiu [1994] for the details of results not explained here.

We start with a (finite-dimensional) symplectic manifold $P$ with symplectic form $\Omega$ and a Lie group $G$ with a Lie algebra $\mathfrak{g}$. We assume that $G$ acts symplectically on $P$ and that it has an equivariant momentum map $\mathbf{J}: P \rightarrow \mathfrak{g}^{*}$, where $\mathfrak{g}^{*}$ is the dual of the vector space $\mathfrak{g}$. Equivariant here means that the map $\mathbf{J}$ is equivariant with respect to the action of $G$ on $P$ and the coadjoint action on $\mathfrak{g}^{*}$. Denoting the relevant actions simply by $g z$ and $g \mu$ respectively, equivariance means that $\mathbf{J}(g z)=g \mathbf{J}(z)$. For concrete examples, such as angular momentum, this just means that the angular momentum "transforms as a vector" under rotations. For each $\xi \in \mathfrak{g}$, we let $J_{\xi}=\langle\mathbf{J}, \xi\rangle$ denote the component of the momentum map along $\xi$.

We also assume that we are given a $G$-invariant Hamiltonian $H$ that describes the dynamics of interest. The associated Hamiltonian vector field is denoted $X_{H}$. Thus, the dynamical equations of motion of interest can be written $\dot{z}=X_{H}(z)$. The vector valued function $\mathbf{J}$ is a constant of the motion along the trajectories of this equation, which may be viewed as one form of Noether's theorem.

We will be considering a relative equilibrium $z_{e} \in P$. This means that there is a Lie algebra element $\xi_{e} \in \mathfrak{g}$ such that $z(t)=\exp \left(\xi_{e} t\right) z_{e}$ for all real numbers $t$, where $z(t)$ denotes the dynamical orbit with initial condition $z_{e}$. We let the value of the momentum at the relative equilibrium be denoted $\mu_{e}=\mathbf{J}\left(z_{e}\right)$ and make the blanket assumption throughout this paper that $z_{e}$ is a regular point of $\mathbf{J}$; that is, $\mathbf{D J}\left(z_{e}\right): T_{z_{e}} P \rightarrow \mathfrak{g}^{*}$, the derivative of $\mathbf{J}$ at $z_{e}$ from $T_{z_{e}} P$, the tangent space to $P$ at the point $z_{e}$, to $\mathfrak{g}^{*}$, is surjective. This assumption is equivalent to the condition that for each nonzero element $\xi$ of the Lie algebra, the corresponding infinitesimal generator evaluated at $z_{e}$, denoted $\xi_{P}\left(z_{e}\right)$, is nonzero.

Although we assume that $z_{e}$ is a regular point, we do not necessarily assume that $\mu_{e}$ is a generic point in $\mathfrak{g}^{*}$. That is, the coadjoint orbit through the point $\mu_{e}$ need not be of maximal dimension. A theorem of Duflo and Vergne (see Marsden and Ratiu [1994, p. 278-9]) states that the generic points form an open and dense set in the dual of the Lie algebra. 
Another way to understand the meaning of "generic" is to introduce the isotropy group $G_{\mu_{e}}$ of $\mu_{e}$, that is, the subgroup of $G$ that leaves the point $\mu_{e}$ invariant under the coadjoint action of $G$ on $\mathfrak{g}^{*}$. A point is generic when its isotropy subgroup is minimal. Thus, nongeneric points will have isotropy subgroups that are "larger than normal".

As is well known for relative equilibria, $H-J_{\xi_{e}}$ has a critical point at $z_{e}$. The energy-momentum method of Simo, Lewis and Marsden [1991] is a method that determines stability by examining definiteness of the second variation of this augmented energy function. These authors concentrate on putting the second variation of $H-J_{\xi_{e}}$ at $z_{e} \in P$ along with the symplectic form into a normal form (with the second derivative of $H-J_{\xi_{e}}$ at $z_{e} \in P$ block diagonal) so that the definiteness can be determined. In this paper we are less concerned with this block diagonal form since we will be able to determine the definiteness using the energy-Casimir method. However, one should be aware that the block diagonal form of the second derivative of the augmented energy together with the normal form for the symplectic structure, is useful for putting the linearized equations at a relative equilibrium into normal form (see Bloch, Krishnaprasad, Marsden and Ratiu [1994, 1995] for the explicit expression for this normal form). Such results may be useful for nonlinear normal form, bifurcation, and control results.

In our examples, the isotropy group need not be compact, and the value of $\mu_{e}$ need not be generic, and it is known (e.g., see Libermann and Marle [1987] and Krishnaprasad [1989]) that one must be careful in such situations with the sense in which one has stability.

\section{$2.2 \quad$ A result of Patrick}

Patrick's theorem rests on the following assumptions.

Assumption 1 The restriction of the coadjoint action of $G_{\mu_{e}}$ on $\mathfrak{g}^{*}$ is proper and there is an inner product on $\mathfrak{g}^{*}$ that is invariant under this action.

Assumption 1 holds, for example, if $G_{\mu_{e}}$ is compact (and this in turn holds if $G$ is compact).

To state the next assumption, we will recall a few facts and establish a little notation. Since we are assuming that $z_{e}$ is a regular point of the momentum map $\mathbf{J}$, it follows that $\operatorname{ker} \mathbf{D J}\left(z_{e}\right)$ is the tangent space to the level set $\mathbf{J}(z)=\mu_{e}$ at the point $z_{e}$. Also, the tangent space to the $G_{\mu_{e}}$-orbit of $z_{e}$ at $z_{e}$ is given by the vector space consisting of infinitesimal generators $\xi_{P}\left(z_{e}\right)$ as $\xi$ ranges over $\mathfrak{g}_{\mu_{e}}$, the Lie algebra of $G_{\mu_{e}}$. Choose a vector subspace $E_{z_{e}} \subset \operatorname{ker} \mathbf{D J}\left(z_{e}\right)$ that complements the tangent space to the $G_{\mu_{e}}$-orbit of $z_{e}$; i.e., $\operatorname{ker} \mathbf{D J}\left(z_{e}\right)=E_{z_{e}} \oplus T_{z_{e}} G_{\mu_{e}}\left(z_{e}\right)$, as in Figure 2.2.1.

Assumption 2 Assume that the second derivative of $H-J_{\xi_{e}}$ at $z_{e} \in P$ restricted to $E_{z_{e}}$ is definite.

Assumption 2 is independent of the choice of complement chosen. The block diagonalization method of Simo, Lewis and Marsden [1991] chooses a particular complement $E_{z_{e}}$ so that the second variation is block diagonal and, simultaneously, that the symplectic form also achieves a certain normal form.

Theorem 2.1 [Patrick, 1992] Under the conditions of Assumptions 1 and 2, the relative equilibrium $z_{e}$ is stable modulo $G_{\mu_{e}}$.

We next explain the meaning of stability modulo a subgroup $K$ of $G$, and in particular, stability modulo $G_{\mu_{e}}$. Consider the orbit $\mathcal{O}=\{z(t) \mid t \geq 0\}$, where $z(t)$ is an integral curve of the vector 


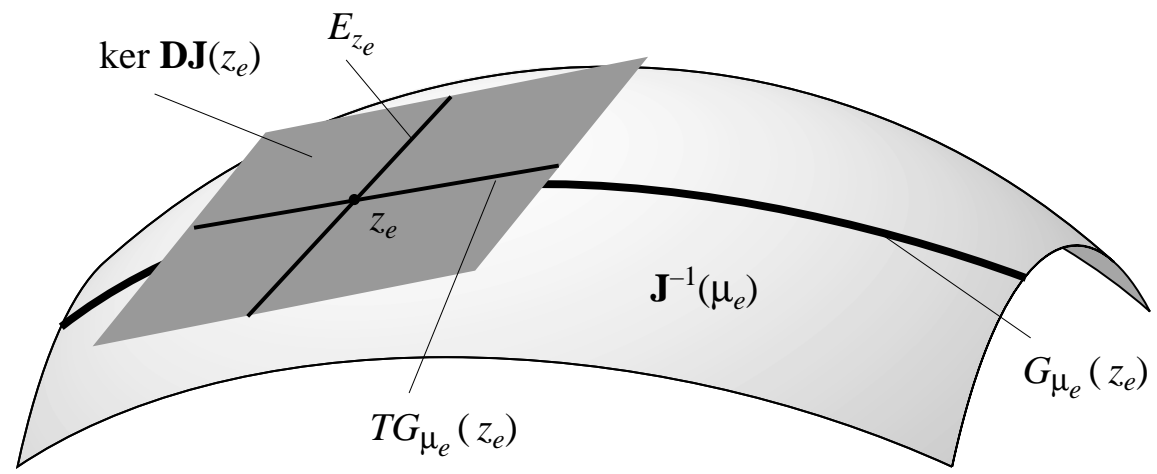

Figure 2.2.1: A space $E_{z_{e}}$ complementary to the group orbit within the level set of the momentum map.

field $X_{H}$. We say $\mathcal{O}$ is stable modulo $K$ provided that for every $K$-invariant open neighborhood $U$ of $\mathcal{O}$, there is an open neighborhood $V$ of $\mathcal{O}$ such that for any initial condition in $V$, its forward trajectory stays in $U$. In the Hamiltonian case, as here, one can, in fact, take the trajectories to be two sided in time, but of course if dissipation is added, one considers the trajectories forward in time only. Intuitively, stability modulo $K$ is the usual notion of Liapunov stability except that one allows arbitrary drift along the orbits of $K$.

The preceding theorem is proved by constructing, with the aid of the invariant metric of Assumption 1 and the conserved energy and momentum, a function that is used as a Liapunov function. The main point to be careful about is how to deal with points with different momentum value than that of the equilibrium, while remembering that the isotropy groups of nearby values of the momentum may, in the nongeneric case, be smaller than that of the relative equilibrium. The invariant metric and the slice theorem for group actions allows one to do this.

Patrick [1995] studies the drift (geometric phase drift) along the group directions; i.e., the drift in the $G_{\mu_{e}}$ directions that are not controlled by the stability theorem. As we shall see, both in theory and simulations, the underwater vehicle problem does have interesting drifts.

\subsection{Relation to the Energy-Casimir Method}

It is useful to specialize the above theorem to the Lie-Poisson context and to relate it to the energyCasimir method. In addition to needing this relation, we will need to generalize the result to allow certain kinds of nonproperness. We do the latter in $\S 3$.

Let $G$ be a Lie group with Lie algebra $\mathfrak{g}$; let $G$ act on itself by left translation and lift this action to the cotangent bundle $T^{*} G$ by cotangent lift. Let $\mathbf{J}: T^{*} G \rightarrow \mathfrak{g}^{*}$ denote the corresponding momentum map, which in this case is simply right translation to the identity. (See for example, Marsden and Ratiu [1994] or Marsden [1992] for the proofs of these statements).

Let $H: T^{*} G \rightarrow \mathbb{R}$ be a $G$-invariant Hamiltonian and let $h: \mathfrak{g}^{*} \rightarrow \mathbb{R}$ be the corresponding function induced on the dual of the Lie algebra. Consider the coadjoint orbit $\mathcal{O}$ through a point $\mu_{e} \in \mathfrak{g}^{*}$ and let, as previously, $G_{\mu_{e}}$ denote the corresponding coadjoint isotropy group. Suppose that $\mu_{e}$ is an equilibrium point for $h$; that is, $\mu_{e}$ is a relative equilibrium for $H$. In other words, $h \mid \mathcal{O}$ has a critical point at $\mu_{e}$.

Let $\xi_{e}=\delta h / \delta \mu$ evaluated at the point $\mu_{e}$. The condition that the restriction of $h$ to $\mathcal{O}$, the 
coadjoint orbit through $\mu_{e}$, have a critical point at $\mu_{e}$ is equivalent to the statement that $\mu_{e}$ is a critical point of $H-J_{\xi_{e}}$, where $\mu_{e} \in \mathfrak{g}^{*} \subset T^{*} G$ is regarded as a point in $T^{*} G$.

Using the preceding comments, we are ready to explain how to relate the energy-Casimir method on $\mathfrak{g}^{*}$ with the energy-momentum method on $T^{*} G$.

We use Assumption 1 with the following modified assumption:

Assumption $2^{\prime}$ The second derivative of $h \mid \mathcal{O}$ at $\mu_{e}$ is definite.

Assumption $2^{\prime}$ is equivalent to Assumption 2. This follows for the present case in which $P=T^{*} G$ because of the following facts. First, the reduced space at $\mu_{e}$ is the coadjoint orbit $\mathcal{O}$ through $\mu_{e}$. Second, the reduced Hamiltonian $h$ is related to the original one $H$ by $H \mid \mathbf{J}^{-1}\left(\mu_{e}\right)=h \circ \pi_{e}$, where $\pi_{e}$ is the projection of $\mathbf{J}^{-1}\left(\mu_{e}\right)$ to the reduced space (in this case, $\pi_{e}$ is right translation to the identity). Third, $\mathbf{J}$ is constant on the level set and the derivative of $\pi_{e}$ maps $E_{z_{e}}$ isomorphically to the tangent space to $\mathcal{O}$ at $\mu_{e}$. Finally, the second derivative of $H-J_{\xi_{e}}$ at $z_{e} \in P$ restricted to $E_{z_{e}}$ is mapped isomorphically to the second derivative of the reduced Hamiltonian $h$ at the point $\mu_{e}$ restricted to $\mathcal{O}$.

An important additional observation concerns the following condition:

Condition $2_{\mathrm{C}}^{\prime}$ There is a function $C$, constant on the orbit $\mathcal{O}$, such that $h+C$ has a critical point at $\mu_{e}$, and the second variation of $h+C$ taken within $\mathfrak{g}^{*}$ at $\mu_{e}$ is definite.

Proposition 2.2 Assumption $2^{\prime}$ is implied by Condition $2_{\mathrm{C}}^{\prime}$.

Proof Since $C$ is constant on the orbit, both $h$ and $h+C$ restricted to the orbit have critical points at $\mu_{e}$. Also, the second variation of the restriction of $C$ to the orbit vanishes; therefore, the second variation of the restriction $h \mid \mathcal{O}$ at $\mu_{e}$ equals the restriction of the second variation of $h+C$ to the tangent space to the orbit at $\mu_{e}$. Thus, if the unconstrained second variation of $h+C$ at $\mu_{e}$ is definite, so is the constrained second variation.

Proposition 2.2 relates our procedure to the energy-Casimir method. In our context, it is important to note that the function $C$ need not be a Casimir function. If the orbit is nongeneric, such functions are sometimes called subcasimirs. See Weinstein [1984] for information on the structure of such functions.

In the energy-Casimir method, one often uses Casimir functions so that $h+C$ is a Liapunov function to show that an equilibrium on $\mathfrak{g}^{*}$ is Liapunov stable. However, in our context, we adopt a more detailed strategy, namely following Patrick, we use properness of the action to obtain the requisite stability in the directions off the orbit and we use Casimir functions (and subcasimir functions for nongeneric equilibria) for stability along the orbit. In the following, this strategy will be important because our groups will have both compact and noncompact subgroups and we need to separate the analysis appropriately for subgroups of each type. As we have mentioned in the introduction, the strategy will be to use the method of reduction by stages.

Let us summarize what we have achieved so far.

Corollary 2.3 Under Assumptions 1 and $2^{\prime}$ (or 1 and $2_{\mathrm{C}}^{\prime}$ ), the point $\mu_{e}$ is Liapunov stable in $\mathfrak{g}^{*}$ with respect to the dynamics of $h$. Moreover, relative to the dynamics of $H$ on $T^{*} G$, the point $\mu_{e}$ is stable modulo the action of $G_{\mu_{e}}$.

Proof As we already pointed out, assumption $2^{\prime}$ implies assumption 2, and so the last statement follows from Theorem 2.1. Stability of $\mu_{e} \in \mathfrak{g}^{*}$ is equivalent to stability of $\mu_{e} \in T^{*} G$ modulo $G$, but this is implied by the stronger statement of stability modulo $G_{\mu_{e}}$. 


\section{$3 \quad$ Stability Theory for Semidirect Products}

In many examples, such as the underwater vehicle where the symmetry group is a semidirect product, the properness assumption (Assumption 1) fails and indeed one sees in numerical simulations (see §4) that the conclusions fail as well. An instructive example along the same lines is also presented in Patrick [1992]. Thus, there is a need for generalizing the stability results given in the preceding section.

Our approach to this problem is to make use of the theory of reduction by stages to separate out the vector space, or the noncompact part of a semidirect product group, and to apply the theory in the preceding section to the part of the symmetry group that is compact. In our examples, the semidirect product groups involved are typified by the Euclidean group and for these groups the method of reduction by stages is appropriate. We therefore begin with some results on the theory of reduction by stages. Although more general results are given in Marsden and Ratiu [1996], we give a self contained exposition specific to the case we need here since it is important for our development. Other results on reduction by stages are given in Sjamaar and Lerman [1991] and Landsman [1995]; however, they do not include the noncompact or nonzero momentum cases we require.

\subsection{Reduction by stages}

In this section, we will explain, in a setting appropriate for this paper, the method of reduction by stages. This method is one in which reduction by a semidirect product can be carried out in two successive steps.

Start with a Lie group that is a semidirect product, $S=G(S)$ where $V$ is a vector space and the Lie group $G$ acts on $V$ (and hence on its dual space $V^{*}$ ). Recall that as sets, $S=G \times V$ and that group multiplication is given by

$$
\left(g_{1}, v_{1}\right) \cdot\left(g_{2}, v_{2}\right)=\left(g_{1} g_{2}, v_{1}+g_{1} v_{2}\right)
$$

where the action of $g \in G$ on $v \in V$ is denoted simply as $g v$. The Lie algebra of $S$ is the semidirect product of Lie algebras: $\mathfrak{s}=\mathfrak{g}(S)$. The bracket is given by

$$
\left[\left(\xi_{1}, v_{1}\right),\left(\xi_{2}, v_{2}\right)\right]=\left(\left[\xi_{1}, \xi_{2}\right], \xi_{1} v_{2}-\xi_{2} v_{1}\right)
$$

where we denote the induced action of $\mathfrak{g}$ on $V$ by concatenation, as in $\xi_{1} v_{2}$.

Later, we will need the formulas for the adjoint and the coadjoint actions for semidirect products. Denoting these and other actions by simple concatenation, they are given as follows (see, e.g., Marsden, Ratiu and Weinstein [1984ab]):

$$
(g, v)(\xi, u)=\left(g \xi, g u-\rho_{v}(g \xi)\right) .
$$

and

$$
(g, v)(\mu, a)=\left(g \mu+\rho_{v}^{*}(g a), g a\right),
$$

where $(g, v) \in S=G \times V,(\xi, u) \in \mathfrak{s}=\mathfrak{g} \times V,(\mu, a) \in \mathfrak{s}^{*}=\mathfrak{g}^{*} \times V^{*}$ and where $\rho_{v}: \mathfrak{g} \rightarrow V$ is the derivative of the map $g \mapsto g v$ at the identity and $\rho_{v}^{*}: V^{*} \rightarrow \mathfrak{g}^{*}$ is its dual. The infinitesimal action of $\mathfrak{g}$ on $V$ will often be denoted by $\xi v$; note that $\xi v=\rho_{v}(\xi)$. 
Next we consider a symplectic action of $S$ on a symplectic manifold $P$ and assume that this action has an equivariant momentum map $\mathbf{J}_{S}: P \rightarrow \mathfrak{s}^{*}$. Since $V$ is a (normal) subgroup of $S$, it also acts on $P$ and has a momentum map $\mathbf{J}_{V}: P \rightarrow V^{*}$ given by

$$
\mathbf{J}_{V}=i_{V}^{*} \circ \mathbf{J}_{S}
$$

where $i_{V}: V \rightarrow \mathfrak{s}$ is the inclusion $v \mapsto(0, v)$ and $i_{V}^{*}: \mathfrak{s}^{*} \rightarrow V^{*}$ is its dual. We think of this merely as saying that $\mathbf{J}_{V}$ is the second component of $\mathbf{J}_{S}$.

We can regard $G$ as a subgroup of $S$ by $g \mapsto(g, 0)$. Thus, $G$ also has a momentum map that is the first component of $\mathbf{J}_{S}$. Equivariance of $\mathbf{J}_{S}$ under $G$ implies the following relation for $\mathbf{J}_{V}$ :

$$
\mathbf{J}_{V}(g z)=g \mathbf{J}_{V}(z)
$$

where we denote the appropriate action of $g \in G$ on an element by concatenation, as before. To prove this formula, one uses the fact that for the coadjoint action of $S$ on $\mathfrak{s}^{*}$, the second component is just the dual of the given action of $G$ on $V$.

We can carry out reduction of $P$ by $S$ at a regular (but not necessarily generic!) value $\sigma=(\mu, a)$ of the momentum map $\mathbf{J}_{S}$ for $S$ in two stages using the following procedure.

- First reduce $P$ by $V$ at the value $a \in V^{*}$ (assume it to be a regular value) to get the reduced space $P_{a}=\mathbf{J}_{V}^{-1}(a) / V$. Here the reduction is by an abelian group, so the quotient is done using the whole of $V$. We will let the projection to the reduced space be denoted $\pi_{a}$ :

$$
\pi_{a}: \mathbf{J}_{V}^{-1}(a) \rightarrow P_{a}
$$

- Form the group $G_{a}$ consisting of elements of $G$ that leave the point $a \in V^{*}$ fixed using the action of $G$ on $V^{*}$. One shows (see the Appendix) that the group $G_{a}$ acts symplectically on $P_{a}$ and has a naturally induced momentum map $\mathbf{J}_{a}: P_{a} \rightarrow \mathfrak{g}_{a}^{*}$, where $\mathfrak{g}_{a}$ is the Lie algebra of $G_{a}$

- Reduce $P_{a}$ at the point $\mu_{a}:=\mu \mid \mathfrak{g}_{a} \in \mathfrak{g}_{a}^{*}$ to get the reduced space $\left(P_{a}\right)_{\mu_{a}}=\mathbf{J}_{a}^{-1}\left(\mu_{a}\right) /\left(G_{a}\right)_{\mu_{a}}$.

The main theorem on reduction by stages for semidirect products, which is proved in the Appendix, can now be stated (see Figure 3.1.1).

Theorem 3.1 Reduction by Stages for Semidirect Products. The reduced space $\left(P_{a}\right)_{\mu_{a}}$ is symplectically diffeomorphic to the reduced space $P_{\sigma}$ obtained by reducing $P$ by $S$ at the point $\sigma=(\mu, a)$.

In the preceding theorem, choose $P=T^{*} S$ where $S=G(S) V$ is a semidirect product as above, with the cotangent action of $S$ on $T^{*} S$ induced by left translations of $S$ on itself. Reducing $T^{*} S$ by the action of $V$ gives a space naturally isomorphic to $T^{*} G$. Thus, the reduction by stages theorem gives as a corollary, the well known semidirect product reduction theorem:

Theorem 3.2 Semidirect Product Reduction. The reduction of $T^{*} G$ by $G_{a}$ at values $\mu_{a}=$ $\mu \mid \mathfrak{g}_{a}$ gives a space that is isomorphic to the coadjoint orbit through the point $\sigma=(\mu, a) \in \mathfrak{s}^{*}=$ $\mathfrak{g}^{*} \times V^{*}$, the dual of the Lie algebra $\mathfrak{s}$ of $S$. 


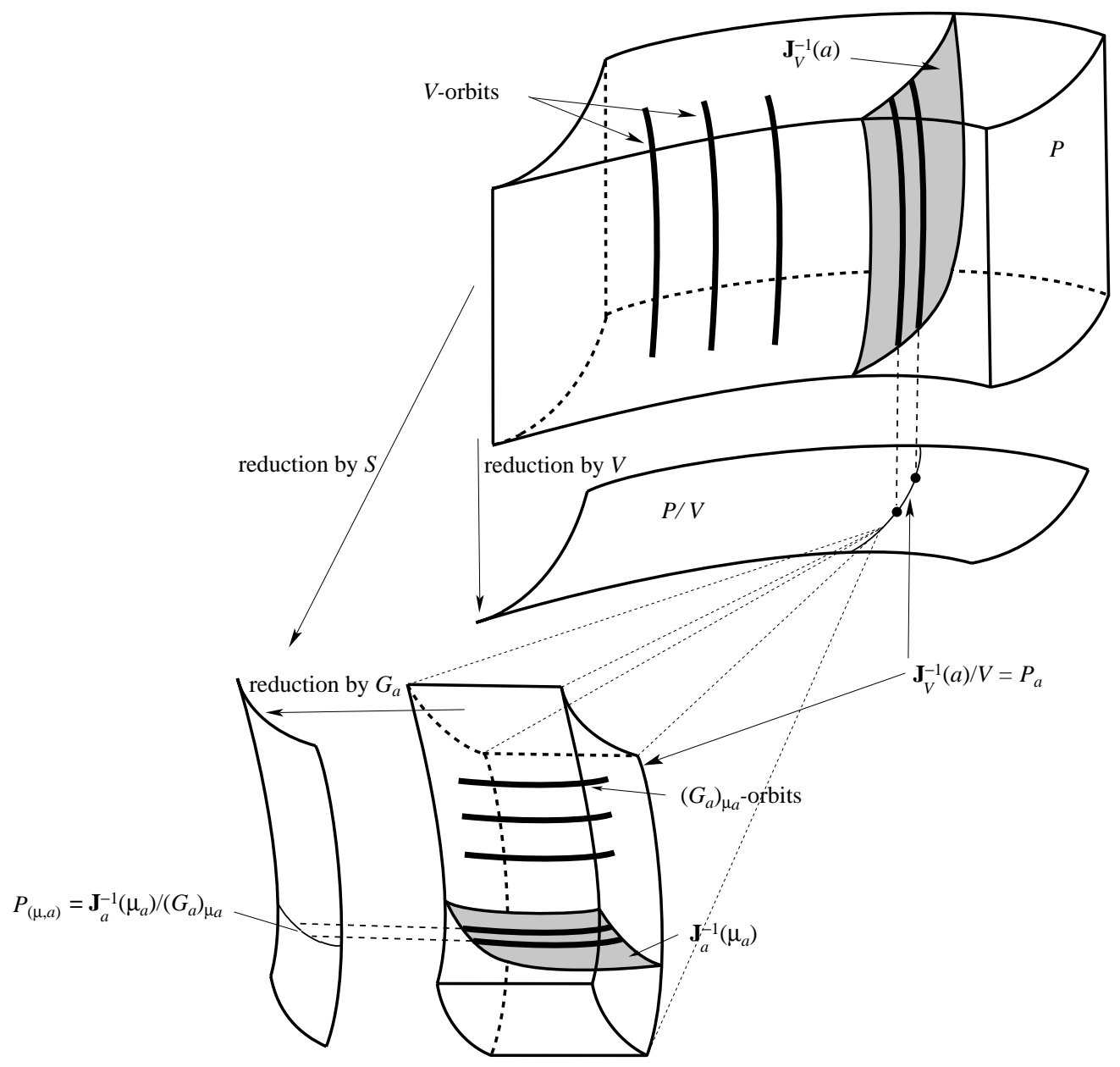

Figure 3.1.1: Reduction by a semidirect product can be achieved in two successive stages.

The original proof of this result given in Marsden, Ratiu and Weinstein [1984ab] requires an essential modification to obtain the more general reduction by stages result, Theorem 3.1.

In the framework of the reduction by stages theorem, one can also reduce the dynamics of a given invariant Hamiltonian in two stages. Reduction by stages allows one to drop the dynamics of a given $S$-invariant Hamiltonian $H$ in two steps. An additional remark is relevant here, namely in many problems, one does not start with a Hamiltonian on $P$, but with one on $P_{a}$. That is, the physical problem may start with a phase space that is recognized to be the result of a first reduction and with a Hamiltonian $H_{a}: P_{a} \rightarrow \mathbb{R}$ that is recognized to be the reduction of an $S$ invariant Hamiltonian $H$ on $T^{*} S$. The condition on $H_{a}$ that guarantees that it be the reduction of an $S$-invariant $H$ is that $H_{g a}([g z])=H_{a}([z])$, where $[z] \in P_{a}$ denotes the equivalence class of $z \in \mathbf{J}_{V}^{-1}(a)$.

For example, for the heavy top (a rigid body with a fixed point and moving in a gravitational field), the physical phase space is $T^{*} S O(3)$ and the symmetry group is $S^{1}$, regarded as rotations about the $z$-axis, the axis of gravity. In this case, one identifies the phase space $T^{*} S O(3)$ with the 
reduction of the cotangent bundle of the special Euclidean group $S E(3)$ by the Euclidean translation subgroup $\mathbb{R}^{3}$ and identifies the symmetry group $S^{1}$ with the isotropy group $G_{a}=S^{1}$ where $a$ is a vector aligned with the direction of gravity and where $S O(3)$ acts on $\mathbb{R}^{3}$ in the standard way. The semidirect product reduction theorem then shows that the reduced space is the reduction of $T^{*} S E(3)$ by the (left) action of $S E(3)$, that is, coadjoint orbits of $S E(3)$.

On the other hand, by the cotangent bundle reduction theorem (see, e.g., Marsden [1992]), the reduction of $T^{*} S O(3)$ by the isotropy group $G_{a}=S^{1}$ is the cotangent bundle of $S^{2}=S O(3) / S^{1}$ with a canonical plus magnetic symplectic structure. The semidirect product reduction point of view is useful because it allows one to use the structure of the dual of the Euclidean Lie algebra for the stability calculations by means of the energy-Casimir method. In our example, we will use a similar strategy, but in a context where the orbits (both generic and nongeneric) are richer than in the dual of the Euclidean Lie algebra.

\subsection{Semidirect Product Stability-Generic Momenta}

Our technique for studying stability is that if $\left(G_{a}\right)_{\mu_{a}}$ is compact (or more generally that the relevant properness and invariant metric hypotheses apply), then we can apply Patrick's result to the second reduction in the method of reduction by stages to obtain stability in the original space $P$ modulo $\left(G_{a}\right)_{\mu_{a}}$ (S) $V$. In this section we will make the assumption that the value of the momentum map is generic. This will be useful, for example, in the case of the underwater vehicle with coincident centers of buoyancy and gravity and nonzero linear momentum. $§ 3.4$ deals with the more general case of nongeneric momentum and it requires an additional hypothesis. In the present case one does not need the full strength of Patrick's theorem.

In the setting of the reduction by stages theorem, let $z_{e} \in P$ be a relative equilibrium for the action of $S$ and for an $S$-invariant Hamiltonian $H$. Let the corresponding values of the momentum map be denoted $\sigma=(\mu, a)$ - we drop the subscripts $e$ on the momenta for simplicity of notation. As above, let $\left[z_{e}\right]=\pi_{a}\left(z_{e}\right) \in P_{a}$ denote the corresponding point in $P_{a}$. Thus, $\left[z_{e}\right]$ is a relative equilibrium in $P_{a}$ for the action of $G_{a}$. We will now apply the previous stability results to this context. Correspondingly, we make the following assumptions.

Assumption $1_{\mathrm{GS}}$ The action of $\left(G_{a}\right)_{\mu_{a}}$ on $\mathfrak{g}_{a}^{*}$ is proper and there is an inner product on $\mathfrak{g}_{a}^{*}$ that is invariant under this action.

Assumption $1_{\mathrm{GS}}$ holds, for example, if $\left(G_{a}\right)_{\mu_{a}}$ is compact (and this holds if $G$ is compact). Analogous to the earlier situation, we choose a vector subspace $E_{\left[z_{e}\right]} \subset \operatorname{ker} \mathbf{D} \mathbf{J}_{a}\left(\left[z_{e}\right]\right)$ that complements the tangent space to the $\left(G_{a}\right)_{\mu_{a}}$-orbit of $\left[z_{e}\right]$; i.e., $\operatorname{ker} \mathbf{D J}_{a}\left(\left[z_{e}\right]\right)=E_{\left[z_{e}\right]} \oplus T_{\left[z_{e}\right]}\left(G_{a}\right)_{\mu_{a}}\left(\left[z_{e}\right]\right)$. Note that $\operatorname{ker} \mathbf{D} \mathbf{J}_{a}\left(\left[z_{e}\right]\right)$ is the tangent space to the level set $\mathbf{J}_{a}([z])=\mu_{a}$ at the point $\left[z_{e}\right]$. Also, the tangent space to the $\left(G_{a}\right)_{\mu_{a}}$-orbit of $\left[z_{e}\right]$ at $\left[z_{e}\right]$ is given by the vector space consisting of infinitesimal generators $\xi_{P}\left(\left[z_{e}\right]\right)$ as $\xi$ ranges over $\left(\mathfrak{g}_{a}\right)_{\mu_{a}}$, the Lie algebra of $\left(G_{a}\right)_{\mu_{a}}$. We let $\xi_{e} \in \mathfrak{g}_{a}$ denote the Lie algebra element corresponding to the relative equilibrium $\left[z_{e}\right]$. Also, we let the Hamiltonian reduced to the first stage space $P_{a}$ be denoted $H_{a}$.

Assumption $2_{\mathrm{GS}}$ The second derivative of $H_{a}-\left(J_{a}\right)_{\xi_{e}}$ at $\left[z_{e}\right] \in P_{a}$ restricted to $E_{\left[z_{e}\right]}$ is definite.

Finally, we assume for this section:

Assumption $3_{\mathrm{GS}}$ The value $\mu_{a}=\left(J_{a}\right)_{\xi_{e}}\left(\left[z_{e}\right]\right)$ is generic in $\mathfrak{g}_{a}^{*}$. 
Theorem 3.3 Let assumptions $1_{\mathrm{GS}}, 2_{\mathrm{GS}}$ and $3_{\mathrm{GS}}$ hold, let $H$ be an $S$-invariant Hamiltonian as above, and let $z_{e} \in P$ be a relative equilibrium for the group $S$. Then the point $\left[z_{e}\right] \in P_{a}$ is Liapunov stable modulo the action of $\left(G_{a}\right)_{\mu_{a}}$ for the dynamics of $H_{a}$ and $z_{e}$ is Liapunov stable in $P$ modulo the action of $\left(G_{a}\right)_{\mu_{a}}$ (S $V$.

Proof The stability of $\left[z_{e}\right] \in P_{a}$ modulo $\left(G_{a}\right)_{\mu_{a}}$ follows from the previous stability theorem in $\S 2$. But stability for the dynamics of $\left[z_{e}\right]$ in $P_{a}$ is equivalent to the stability of $z_{e}$ in $\mathbf{J}_{V}^{-1}(a)$ modulo $\left(G_{a}\right)_{\mu_{a}}$ (S) $V$. We complete the proof by noting that we can extend this statement to a whole neighborhood of $z_{e}$ in $P$ (that is, we can extend it to nearby level sets) because we are assuming that $a$ is a regular value of $\mathbf{J}_{V}$ and, because $V$ is abelian, the quotient on this upper level is by a fixed group $V$. We also note that the reduced spaces $\left(P_{a}\right)_{\mu_{a}}$ are locally diffeomorphic as the value of $\mu_{a}$ is changed since we are assuming that $\mu_{a}$ is a generic point. Thus, the stability results on the single reduced space extend to a whole neighborhood of $\left[z_{e}\right]$.

We can verify the hypothesis $2_{\mathrm{GS}}$ by using an argument on the Poisson reduced space $P / S$ as we did earlier with the energy-Casimir argument. Namely,

Proposition 3.4 Let $h: P / S \rightarrow \mathbb{R}$ denote the Poisson reduced Hamiltonian and let $\left[\left[z_{e}\right]\right] \in P / S$ denote the Poisson reduced relative equilibrium. Hypothesis $2_{\mathrm{GS}}$ holds if there is a function $C$ : $P / S \rightarrow \mathbb{R}$ constant on the symplectic leaf (that is, the symplectic reduced space obtained by reducing $P$ by the action of $S)$ through $\left[\left[z_{e}\right]\right]$ such that $h+C$ has a critical point at $\left[\left[z_{e}\right]\right]$ and such that the second variation of $h+C$ evaluated at $\left[\left[z_{e}\right]\right]$ is definite.

This follows by an argument analogous to that we gave in the case $P=T^{*} G$ earlier. In this generic case, one normally would use Casimir functions here. In $\S 3.4$ we will use Assumption $22_{\mathrm{GS}}$ for nongeneric equilibria. In that case, $C$ may also be a function of subcasimirs.

\subsection{A Double Semidirect Product Stability Theorem}

There is an additional consequence of Theorem 3.3 that will prove to be useful for the underwater vehicle with noncoincident centers of buoyancy and gravity. This is the double semidirect product case in which not only is the symmetry group a semidirect product (as in §3.2) but also reduction by this symmetry group is described by semidirect product reduction (Theorem 3.2). Let $G$ be a Lie group, $V$ a vector space, and suppose that $G$ acts on $V$. We start with the phase space of a mechanical system given by $P_{\text {mech }}=T^{*} \bar{G}$, where, for notational convenience, we let $\bar{G}=G$ (S) $V$

Next, we consider a double semidirect product of the form $W=G(S)(V \times V)$, where the action of $G$ on $V \times V$ is defined to be $g \cdot(v, u)=(g v, g u)$. One checks that $W=\bar{G}$ (S) $V$, where the action of $\bar{G}$ on $V$ is defined to be $(g, v) \cdot u=g u$. Let $P=T^{*} W$ and notice that $V$ acts on $W$ by translation in the last factor and hence on $P$; this action has a momentum map $\mathbf{J}_{V}: P \rightarrow V^{*}$. The space $P_{\text {mech }}$ is isomorphic to the reduced space associated with this momentum map. We also need to recall (by the Lie-Poisson reduction theorem) that the reduced space $P / W$ is isomorphic to the dual of the Lie algebra of $W$, i.e., $\mathfrak{w}^{*}=\mathfrak{g}^{*} \times V^{*} \times V^{*}$.

Now consider again the phase space $P_{\text {mech }}=T^{*} \bar{G}$. Following the semidirect product reduction theorem, we compute $\bar{G}_{a_{2}}=G_{a_{2}}$ (S) $V$ where $a_{2} \in V^{*}$. Then reduction of $T^{*} \bar{G}$ by $\bar{G}_{a_{2}}$ at values $\left(\mu, a_{1}\right) \mid \overline{\mathfrak{g}}_{a_{2}} \in \overline{\mathfrak{g}}_{a_{2}}^{*}$ gives a space isomorphic to the coadjoint orbit through $\left(\mu, a_{1}, a_{2}\right) \in \overline{\mathfrak{g}}^{*} \times V^{*}=\mathfrak{w}^{*}$. 
Now suppose that we have Hamiltonian dynamics on $P_{\text {mech }}$ given by a $\bar{G}_{a_{2}}$-invariant Hamiltonian $H_{\text {mech }}$. Suppose further that $H_{\text {mech }}$ is the reduction of a $W$-invariant Hamiltonian $H$ on $P$. We denote the reduced Hamiltonian on $\mathfrak{w}^{*}$ by $h$. For $z_{\text {emech }} \in P_{\text {mech }}$ a relative equilibrium, we let $z_{e}$ be the corresponding equilibrium in $P$ and $\left[\left[z_{e}\right]\right]=\omega_{e}=\left(\mu, a_{1}, a_{2}\right) \in \mathfrak{w}^{*}$ the reduced relative equilibrium.

We can apply the preceding proposition to this setup with the following assumptions:

Assumption $1_{\mathrm{DSDP}}$ Let $a=\left(a_{1}, a_{2}\right) \in V^{*} \times V^{*}$. Assume that the action of $\left(G_{a}\right)_{\mu_{a}}$ on $\mathfrak{g}_{a}^{*}$ is proper and there is an inner product on $\mathfrak{g}_{a}^{*}$ that is invariant under this action.

Assumption $1_{\mathrm{DSDP}}$ holds, for example, if $\left(G_{a}\right)_{\mu_{a}}$ is compact (and this holds if $G$ is compact).

Assumption 2 DSDP Consider the coadjoint orbit $\mathcal{O} \subset \mathfrak{g}^{*} \times V^{*} \times V^{*}$ through the point $\omega_{e}=$ $\left(\mu, a_{1}, a_{2}\right)$. Assume that there is a function $C$ constant on $\mathcal{O}$ such that $h+C$ has a critical point at $\omega_{e}$ and that its second variation at this point is definite.

Assumption $3_{\mathrm{DSDP}}$ The point $\omega_{e}=\left(\mu, a_{1}, a_{2}\right)$ is a generic point in $\mathfrak{g}^{*} \times V^{*} \times V^{*}$.

Theorem 3.5 Let the preceding assumptions $1_{\mathrm{DSDP}}, 2_{\mathrm{DSDP}}$ and $3_{\mathrm{DSDP}}$ hold. Then the point $z_{\text {emech }} \in P_{\text {mech }}$ is Liapunov stable modulo the action of $\left(G_{a}\right)_{\mu_{a}}$ (S $V$ for the dynamics of $H_{\text {mech }}$. Moreover, $\omega_{e}$ is a stable point of the Lie-Poisson dynamics on $\mathfrak{w}^{*}$.

Proof From Theorem 3.3 and Proposition 3.4 applied to $P=T^{*}(G \subseteq(V \times V))$, it follows that $z_{e} \in P$ is stable modulo $\left(G_{a}\right)_{\mu_{a}}$ (S $(V \times V)=\left(\left(G_{a}\right)_{\mu_{a}}\right.$ (S $\left.V\right)$ (S) $V$. But, stability for the dynamics of $z_{e}$ in $P$ modulo $\left(\left(G_{a}\right)_{\mu_{a}}\right.$ (S $\left.V\right)$ (S $V$ is equivalent to stability of $z_{e \text { mech }}$ in $\mathbf{J}_{V}^{-1}\left(a_{2}\right) / V$ modulo $\left(G_{a}\right)_{\mu_{a}}$ (S) $V$

\subsection{Semidirect Product Stability-Nongeneric Momenta}

Consider again the setup of $\S 3.2$. When the momentum value of a relative equilibrium is nongeneric, as in the case of a rising, bottom-heavy underwater vehicle, we can proceed in two ways. First, we can restrict our attention to a single reduced space (a single coadjoint orbit). The results above would then apply, without the third assumption. We call this phenomenon leafwise stability. However, this need not imply stability for values of the momentum close to but not equal to $a$ since the dimension of the reduced space and the stability properties on it may change as the momentum is varied. An example showing that some additional hypothesis is needed is the following:

Example (Patrick). Consider the dual of the Lie algebra of the Euclidean group in the plane, $\mathfrak{s e}(2)^{*}$ with its standard Lie-Poisson structure. Identify this space (see Marsden and Ratiu [1994] for details) with $\mathbb{R}^{3}$. Coadjoint orbits consist of cylinders centered on the $z$-axis with nonzero radius and single points on the $z$-axis. Each point on the $z$-axis is a relative equilibrium for any Hamiltonian and also each such point is obviously leafwise stable since it is a single point. However, such points need not be stable in $\mathfrak{s e}(2)^{*}$ as the following smooth Hamiltonian written in cylindrical coordinates shows: $H(z, r, \phi)=r \cos \phi$. The instability arises from a slow drift in the $z$-direction as is seen from the equations of motion for this Hamiltonian. The hypotheses introduced later in this section exclude this Hamiltonian. 
The second way to proceed is to find additional sufficient conditions to guarantee that leafwise stability extends to stability. We provide sufficient conditions that are relevant to some relative equilibria of the underwater vehicle. As we shall point out, there are some places where additional theoretical work is needed.

For the rising/spinning, bottom-heavy underwater vehicle one has a nongeneric value of the momentum. Fortunately, the conditions for leafwise stability occur in a regime for which our conditions below are satisfied (as we shall see) and so one in fact gets stability. As we shall also see, the failure of the stability conditions corresponds precisely to interesting critical phenomena in the spectrum of the linearized equations: each critical spectral event in this example (passing of eigenvalues, splitting of eigenvalues) corresponds to an interesting event in the energy-momentum stability analysis.

To extend leafwise stability to stability, the critical issue is to control the momentum variables in the new "noncompact directions" that may arise in the reduced space when we shift from the given equilibrium with a nongeneric momentum value to a nearby generic one. By the arguments used to prove Patrick's theorem, we do not need to worry about any other new "compact directions" that may arise.

The main point involved can be seen using the formula for the coadjoint action for a semidirect product given earlier:

$$
(g, v)(\mu, a)=\left(g \mu+\rho_{v}^{*}(g a), g a\right) .
$$

The "compact part" of the group action corresponds to the action of $G$ while the "noncompact part" corresponds to the action of $V$. As we change $(\mu, a)$ from a nongeneric to a generic point, the orbit for this action can change dimension; the part we are concerned with is the change due to the noncompact part. This change is thus given by the difference between the sets

$$
E:=\left\{\left(\rho_{v}^{*}(a), 0\right) \mid v \in V\right\} \subset \mathfrak{g}^{*} \times V
$$

at our equilibrium value of the momentum $a$ and the corresponding set for other values $a^{\prime}$ near $a$ :

$$
E^{\prime}=\left\{\left(\rho_{v}^{*}\left(a^{\prime}\right), 0\right) \mid v \in V\right\} \subset \mathfrak{g}^{*} \times V .
$$

Thus, we are concerned with a complement to the linear space $E$ within $E^{\prime}$. Let such a complement be denoted $F$; that is, the vector space $F$ should be such that

1. for all generic $a^{\prime}$ in a neighborhood of $a$, we have $E \oplus F=E^{\prime}$ and

2. for all $a^{\prime}$ in a neighborhood, generic or not, $E \oplus F \supset E^{\prime}$.

For example, if $a=0$ and if $E^{\prime}=\mathfrak{g}$, then we may choose $F=\mathfrak{g}^{*} \times\{0\}$.

Assumption $3_{\mathrm{NG}}$ For some dynamically invariant function (usually Casimir functions and other conserved quantities) $C$, and choice of complement $F$ satisfying the above two conditions, the function $h+C$ has a partial derivative at the nongeneric equilibrium in the direction $F$ equal to zero and its second variation in this direction at the equilibrium is positive definite.

\section{Remarks.}

1. Using the description of reduced spaces as $\mathbf{J}^{-1}(\mathcal{O}) / S$, one can also phrase this condition in terms of the unreduced geometry, in the same manner as we have related the energy momentum method to the energy Casimir method. 
2. If at the nongeneric equilibrium, $h+C$ has a critical point for the entire dual of the Lie algebra $\left(\mathfrak{g}(\mathrm{S} V)^{*}\right.$ and if the second variation is definite, then Assumption $3_{\mathrm{NG}}$ holds. As we shall see, for the rising and spinning, bottom-heavy underwater vehicle, the transition from this condition to the more delicate condition in Assumption $3_{\mathrm{NG}}$ signals an interesting spectral event.

Theorem 3.6 Let assumptions $1_{\mathrm{GS}}, 2_{\mathrm{GS}}$ and $3_{\mathrm{NG}}$ hold, let $H$ be an $S$-invariant Hamiltonian and let $z_{e} \in P$ be a relative equilibrium for the group $S$. Then the point $z_{e}$ is Liapunov stable in $P$ modulo the action of $\left(G_{a}\right)_{\mu_{a}}$ (S $V$.

Proof We obtain the required stability by constructing a Liapunov function obtained by adding together, in the original unreduced phase space, the function of Patrick (controlling the extra compact directions), the function of the above condition $3_{\mathrm{NG}}$, which controls the extra noncompact momentum directions and the energy-momentum function. This total function is then used as in Patrick [1992] to give stability of the relative equilibrium orbit modulo the action of $\left(G_{a}\right)_{\mu_{a}}$ (S) $V$.

A variant of the above theorem is to use the following:

Assumption $3_{\mathrm{NG}}^{\prime}$ For some dynamically invariant function (usually a Casimir function and other conserved quantities) $C$, and choice of complement $F$ satisfying the above two conditions, we have: the space $F$ is one dimensional and the kernel of the derivative of the function $h+C$, evaluated at the nongeneric equilibrium, is transverse to $F$.

Theorem 3.7 Let assumptions $1_{\mathrm{GS}}, 2_{\mathrm{GS}}$ and $3_{\mathrm{NG}}^{\prime}$ hold, let $H$ be an $S$-invariant Hamiltonian and let $z_{e} \in P$ be a relative equilibrium for the group $S$. Then the point $z_{e}$ is Liapunov stable in $P$ modulo the action of $\left(G_{a}\right)_{\mu_{a}}$ (S $V$.

Given the double semidirect product setup of $\S 3.3$, the stability analysis of nongeneric equilibria is extended analogously using Assumption $3_{\mathrm{NG}}$ or $3_{\mathrm{NG}}^{\prime}$.

Corollary 3.8 Let assumptions $1_{\mathrm{DSDP}}$, $2_{\mathrm{DSDP}}$ with $3_{\mathrm{NG}}$ or $3_{\mathrm{NG}}^{\prime}$ hold. Then the point $z_{\text {emech }} \in$ $P_{\text {mech }}$ is Liapunov stable modulo the action of $\left(G_{a}\right)_{\mu_{a}}$ (S) $V$ for the dynamics of $H_{\text {mech }}$. Moreover, $\omega_{e}$ is a stable point of the Lie-Poisson dynamics on $\mathfrak{w}^{*}$.

The proof is analogous to the proof of Theorem 3.5 except that Theorem 3.6 or Theorem 3.7 is used in place of Theorem 3.3.

\section{Remarks.}

1. If $F$ is two dimensional one can seek two conserved functions whose level sets are transverse to $F$ and to each other. In this case, one can use strips bounded by these level sets to trap orbits in neighborhoods in $F$. This type of approach may be useful in the nongeneric case of an underwater vehicle with spin only in three-dimensional space since $F$ has dimension greater than one. However, in this example, if there is an axis of symmetry, one can use Theorem 3.6 (and the remark that precedes it about $h+C$ ). 
2. For the planar underwater vehicle, $F$ is one dimensional, and a direct analysis that uses the integrability of the dynamics confirms what one finds by Theorem 3.7. The rising and spinning bottom-heavy underwater vehicle in three-dimensional space with no axis of symmetry is another example where Assumption $3_{\mathrm{NG}}^{\prime}$ may be used.

\section{Dynamics of an Underwater Vehicle}

In this section, we apply the theorems of $\S 3.2, \S 3.3$ and $\S 3.4$ to determine the stability of relative equilibria for the dynamics of underwater vehicles.

\subsection{Review of the Lie-Poisson form of the Dynamics}

In Leonard [1995b] it was shown that the dynamics of the underwater vehicle problem can be viewed as Lie-Poisson dynamics in the following way. We assume the underwater vehicle is a neutrally buoyant, rigid body submerged in an infinitely large volume of incompressible, inviscid, irrotational fluid which is at rest at infinity. The dynamics of the body-fluid system are described by Kirchhoff's equations, where we assume the only external forces and torques acting on the system are due to buoyancy and gravity. Consider the group $W$, the semidirect product of $S O(3)$ with two copies of $\mathbb{R}^{3}$, i.e., $W=S O(3) \subseteq\left(\mathbb{R}^{3} \times \mathbb{R}^{3}\right)$, where we take the action of $S O(3)$ on $\mathbb{R}^{3} \times \mathbb{R}^{3}$ to be $R \cdot(b, w)=(R b, R w)$. If we further let the action of $S E(3)=S O(3) \subseteq \mathbb{R}^{3}$ on $\mathbb{R}^{3}$ be $(R, b) \cdot w=R w$ then we also have that $W=\left(S O(3) \subseteq \mathbb{R}^{3}\right)$ (S $\mathbb{R}^{3}$. Group multiplication in $W$ is defined for $(R, b, w),\left(R^{\prime}, b^{\prime}, w^{\prime}\right) \in W$ by

$$
(R, b, w)\left(R^{\prime}, b^{\prime}, w^{\prime}\right)=\left(R R^{\prime}, R b^{\prime}+b, R w^{\prime}+w\right) .
$$

Note that $\mathfrak{w}^{*}=\mathfrak{s o}(3)^{*} \times \mathbb{R}^{3^{*}} \times \mathbb{R}^{3^{*}}$ is nine dimensional. Because we allow for the possibility that the vehicle's center of buoyancy may not be coincident with its center of gravity, the underwater vehicle dynamics has Lie-Poisson form on $\mathfrak{w}^{*}$. The generic orbit in $\mathfrak{w}^{*}$ is six dimensional; all of the orbits are calculated explicitly in Leonard [1995b].

Let $(\Pi, \mathrm{P}, \Gamma)$ be an element in $\mathfrak{w}^{*}$. For the underwater vehicle, $\Pi$ and $\mathrm{P}$ correspond, respectively, to angular and linear components of the impulse of the system (roughly, the momentum of the system less a term at infinity). The vector $\Gamma$ describes the direction of gravity in body-fixed coordinates. The Poisson bracket on $\mathfrak{w}^{*}$ is

$$
\{F, K\}(\Pi, \mathrm{P}, \Gamma)=\nabla F^{T} \Lambda(\Pi, \mathrm{P}, \Gamma) \nabla K
$$

where $F$ and $K$ are smooth functions on $\mathfrak{w}^{*}$ and the Poisson tensor $\Lambda$ is given by

$$
\Lambda(\Pi, \mathrm{P}, \Gamma)=\left(\begin{array}{ccc}
\hat{\Pi} & \hat{\mathrm{P}} & \hat{\Gamma} \\
\hat{\mathrm{P}} & 0 & 0 \\
\hat{\Gamma} & 0 & 0
\end{array}\right) .
$$

In this equation, ${ }^{\wedge}: \mathbb{R}^{3} \rightarrow \mathfrak{s o}(3)$ is the standard isomorphism of $\mathbb{R}^{3}$ with the Lie algebra of the rotation group and is defined by $\hat{\alpha} \beta=\alpha \times \beta$ for $\alpha, \beta \in \mathbb{R}^{3}$. Let $\Omega$ be the angular velocity of the vehicle about the center of buoyancy and $v$ be the linear velocity of the center of buoyancy, both vectors expressed with respect to body coordinates. Then,

$$
\begin{aligned}
& \Pi=I \Omega+D v \\
& \mathrm{P}=M v+D^{T} \Omega
\end{aligned}
$$


or equivalently

$$
\begin{aligned}
\Omega & =A \Pi+B^{T} \mathrm{P} \\
v & =C \mathrm{P}+B \Pi,
\end{aligned}
$$

where

$$
\begin{aligned}
& A=\left(I-D M^{-1} D^{T}\right)^{-1} \\
& B=-C D^{T} I^{-1}=-M^{-1} D^{T} A \\
& C=\left(M-D^{T} I^{-1} D\right)^{-1} .
\end{aligned}
$$

$I$ is the matrix that is the sum of the body inertia matrix plus the added inertia matrix associated with the potential flow model of the fluid. Similarly, $M$ is the sum of the mass matrix for the body alone, i.e., the mass of the body $m$ multiplied by the identity matrix, plus the added mass matrix associated with the fluid (note that $M$ itself is not a multiple of the identity unless the body is symmetric). The matrix $D$ accounts for cross terms. In the examples, we will consider the case in which the rigid body has three mutually perpendicular planes of symmetry, i.e., an ellipsoidal body, and with the appropriate choice of body-fixed coordinate frame (i.e., putting the origin of the frame at the vehicle's center of buoyancy and choosing the axes along the principal axes of the displaced fluid), $M$ and $I$ are diagonal and $D=m \hat{r}_{G}$. Here, $r_{G}$ is the vector from the center of buoyancy to the center of gravity.

With these definitions, the underwater vehicle dynamics has Lie-Poisson form on $\mathfrak{w}^{*}$ determined by the Hamiltonian

$$
h(\Pi, \mathrm{P}, \Gamma)=\frac{1}{2}\left(\Pi^{T} A \Pi+2 \Pi^{T} B^{T} \mathrm{P}+\mathrm{P}^{T} C \mathrm{P}-2 m g\left(\Gamma \cdot r_{G}\right)\right) .
$$

Let $\omega=(\Pi, \mathrm{P}, \Gamma) \in \mathfrak{w}^{*}$. Then, the equations of motion are given by $\dot{\omega}_{i}=\left\{\omega_{i}, h\right\}$ or, equivalently,

$$
\dot{\omega}=\Lambda(\omega) \nabla h(\omega) .
$$

These equations of motion can be computed to be

$$
\begin{aligned}
\dot{\Pi} & =\Pi \times \Omega+\mathrm{P} \times v-m g \Gamma \times r_{G} \\
\dot{\mathrm{P}} & =\mathrm{P} \times \Omega \\
\dot{\Gamma} & =\Gamma \times \Omega .
\end{aligned}
$$

A point $\omega=(\Pi, \mathrm{P}, \Gamma) \in \mathfrak{w}^{*}$ is generic if the coadjoint orbit through that point has maximal dimension six. Equivalently, $\omega$ is generic if the Poisson tensor $\Lambda$ has maximal rank six when evaluated at $\omega$. Considering the form (4.1.1) of $\Lambda, \omega$ is generic if and only if $\mathrm{P} \nVdash \Gamma$ (which implies $\mathrm{P} \neq 0$ and $\Gamma \neq 0)$. For generic points, the following are three independent Casimir functions:

$$
C_{1}(\Pi, \mathrm{P}, \Gamma)=\mathrm{P} \cdot \Gamma, \quad C_{2}(\Pi, \mathrm{P}, \Gamma)=\|\mathrm{P}\|^{2}, \quad C_{3}(\Pi, \mathrm{P}, \Gamma)=\|\Gamma\|^{2} .
$$

These are functions which Poisson commute with any function $K$ on $\mathfrak{w}^{*}$, i.e., $\left\{C_{i}, K\right\}=0$. In particular, take $K=h$ to see that Casimir functions are conserved quantities along the equations of motion. 
A point $\omega=(\Pi, \mathrm{P}, \Gamma) \in \mathfrak{w}^{*}$ is nongeneric if $\mathrm{P} \| \Gamma$. In the case that $\mathrm{P}$ and $\Gamma$ are not both zero, then the rank of $\Lambda(\omega)$ is four and the coadjoint orbit through $\omega$ has dimension four. Besides the three Casimirs defined above, two additional conserved quantities on the nongeneric coadjoint orbits are

$$
C_{4}(\Pi, \mathrm{P}, \Gamma)=\Pi \cdot \mathrm{P}, \quad C_{5}(\Pi, \mathrm{P}, \Gamma)=\Pi \cdot \Gamma .
$$

These conserved quantities are subcasimirs. If $\mathrm{P}=\Gamma=0$ with $\Pi \neq 0$, then the rank of $\Lambda(\omega)$ is two and the coadjoint orbit through $\omega$ has dimension two. An additional subcasimir on this nongeneric coadjoint orbit is

$$
C_{6}(\Pi, \mathrm{P}, \Gamma)=\|\Pi\|^{2} .
$$

The underwater vehicle in the case that the center of buoyancy and the center of gravity are coincident, i.e., $r_{G}=0$, was studied as a special case in Leonard [1995b]. For this case, consider the group $S E(3)$, i.e., the semidirect product of $S O(3)$ with only one copy of $\mathbb{R}^{3}$. The dynamics of the underwater vehicle with coincident centers has Lie-Poisson form on $\mathfrak{s e}(3)^{*}$ (which is six dimensional). The generic orbit in $\mathfrak{s e}(3)^{*}$ is four dimensional. Here $\Gamma$ plays no role in the dynamics and $(\Pi, \mathrm{P})$ describes an element in $\mathfrak{s e}(3)^{*}$. The Poisson tensor on $\mathfrak{s e}(3)$ is given by

$$
\Lambda(\Pi, \mathrm{P})=\left(\begin{array}{cc}
\hat{\Pi} & \hat{\mathrm{P}} \\
\hat{\mathrm{P}} & 0
\end{array}\right)
$$

The Hamiltonian describing the dynamics is

$$
h(\Pi, \mathrm{P})=\frac{1}{2}\left(\Pi^{T} A \Pi+2 \Pi^{T} B^{T} P+\mathrm{P}^{T} C \mathrm{P}\right)
$$

with $B=D=0$ if the vehicle is ellipsoidal. The equations of motion are

$$
\begin{aligned}
\dot{\Pi} & =\Pi \times \Omega+\mathrm{P} \times v \\
\dot{\mathrm{P}} & =\mathrm{P} \times \Omega .
\end{aligned}
$$

All points $(\Pi, \mathrm{P}) \in \mathfrak{s e}(3)^{*}$ for which $\mathrm{P} \neq 0$ are generic. Two independent Casimirs are

$$
C_{1}(\Pi, \mathrm{P})=\Pi \cdot \mathrm{P}, \quad C_{2}(\Pi, \mathrm{P})=\|\mathrm{P}\|^{2} .
$$

This is the standard Lie-Poisson description of Kirchhoff's equations for a rigid body in a fluid with no external forces or torques, as can be found, for example, in Arnold [1993]. In the case that $\mathrm{P}=0$, the point $(\Pi, 0) \in \mathfrak{s e}(3)^{*}$ is nongeneric, the orbit through this point is two-dimensional and a subcasimir is $C_{3}(\Pi, \mathrm{P})=\|\Pi\|^{2}$.

\subsection{Dynamics on the Physical Phase Space}

Using the semidirect product theory recalled earlier, one can view the Lie-Poisson dynamics described in the previous section as reduced dynamics starting from either of two places.

First, one can start with $W$ itself and consider the left invariant Hamiltonian on $T^{*} W$ whose value at the identity is given by (4.1.2). Then, the Lie-Poisson reduction theorem (see Marsden and Ratiu [1994]) gives the reduced dynamics on the coadjoint orbits in $\mathfrak{w}^{*}$.

Second, one can start, as in Leonard [1995b], with the (more physical) group $S E(3)$ as the starting configuration space, which represents the literal configurations of the underwater vehicle 
and the same Lagrangian or Hamiltonian, but which now is only invariant under translations and rotations about the directions of gravity. That is, the symmetry group is not the whole group of rotations and translations $S E(3)$, but rather the subgroup $S E(2) \times \mathbb{R}$. This breaking of symmetry is due to the noncoincidence of the center of gravity and the center of buoyancy, as explained in Leonard [1995b]. From this starting point, it is semidirect product reduction, Theorem 3.2, that gives the reduced dynamics on the coadjoint orbits in $\mathfrak{w}^{*}$.

In the case that the centers of buoyancy and gravity are coincident, the two starting points are the same, i.e., one starts with $S E(3)$ and the left-invariant Hamiltonian on $T^{*} S E(3)$ given by (4.1.4). Since gravity plays no role, the symmetry group is $S E(3)$. The Lie-Poisson reduction theorem gives the reduced dynamics on the coadjoint orbits in $\mathfrak{s e}(3)^{*}$.

\subsection{Stability of Generic Momentum Values}

In this section we apply the stability theorems 3.3 and 3.5 to the generic equilibria of the underwater vehicle studied in Leonard [1995b]. The purpose is to show that for the same stability conditions derived in Leonard [1995b], the nature of stability is better than that predicted by the energyCasimir method alone. Simulation results given below indicate that this extended stability is now the best we can expect.

We note that in each of the cases studied below, our extension of Patrick's result using reduction by stages is essential. That is, our examples fail to satisfy the conditions of Patrick's theorem, in particular, Assumption 1 (used in Theorem 2.1) does not hold. This is a result of the fact that for our examples, the symmetry group is not compact.

\subsubsection{Generic Equilibria for a Vehicle with Coincident Centers}

The first case we examine is the vehicle with coincident centers of buoyancy and gravity, which, as was shown in $\S 4.1$, has dynamics that are Lie-Poisson on $\mathfrak{s e}(3)^{*}$. Here the Poisson manifold is $P=T^{*} S E(3)$ and the symmetry group is $S=S E(3)$. Following the notation of $\S 3.1, S=G$ (S) $V$ where $G=S O(3)$ and $V=\mathbb{R}^{3}$. The Poisson-reduced space is $P / S=\mathfrak{s e}(3)^{*}$ and the Poissonreduced Hamiltonian is $h$ as given by (4.1.4). Let $z_{e} \in T^{*} S E(3)$ be a relative equilibrium for the action of $S$ and $\left[\left[z_{e}\right]\right] \in \mathfrak{s e}(3)^{*}$ the Poisson-reduced relative equilibrium. Following the notation of $\S 3.2,\left[\left[z_{e}\right]\right]=\sigma=(\mu, a)$ where $\mu \in \mathfrak{s o}(3)^{*}$ and $a \in \mathbb{R}^{3^{*}}$.

Consider the two-parameter family of equilibrium solutions

$$
\mu=\left(0,0, \Pi_{3}^{0}\right)^{T}, \quad a=\left(0,0, P_{3}^{0}\right)^{T}
$$

which corresponds to steady translation along and rotation about one of the principal axes of the body. As long as $P_{3}^{0} \neq 0$, this relative equilibrium is generic. Let the vehicle be ellipsoidal with inertia matrix $I=\operatorname{diag}\left(I_{1}, I_{2}, I_{3}\right)$ and mass matrix $M=\operatorname{diag}\left(m_{1}, m_{2}, m_{3}\right)$. Further, suppose that the vehicle is symmetric about the axis of rotation, i.e., $I_{1}=I_{2}$ and $m_{1}=m_{2}$. Leonard [1995b] showed that Assumption $2_{\text {GS }}$ is satisfied following the arguments of Proposition 3.4 if and only if

$$
\left(\frac{\Pi_{3}^{0}}{P_{3}^{0}}\right)^{2}>4 I_{2}\left(\frac{1}{m_{3}}-\frac{1}{m_{2}}\right) .
$$

Each $m_{i}$ is inversely related to the length of the ellipsoid's semiaxis $l_{i}$ along the $i$ th principle axis. Thus, condition (4.3.2) is always met when the axis of rotation/translation $l_{3}$ is the shortest axis, 
since this implies $m_{3}>m_{2}$. When $l_{3}$ is the longest axis, then $m_{3}<m_{2}$. In this case, in order to meet condition (4.3.2), the vehicle needs to be spinning sufficiently fast for a given translation. By the energy-Casimir method, if (4.3.2) is met, then $z_{e}$ is stable in $T^{*} S E(3)$ modulo $S E(3)$.

Using the extended stability theorem 3.3 , we can say more about the stability of $z_{e}$.

Theorem 4.1 If (4.3.2) is met, then $z_{e}$ as defined by (4.3.1) is stable in $T^{*} S E(3)$ modulo $S E(2) \times$ $\mathbb{R}$.

Proof Since $G=S O(3)$ is compact, Assumption $1_{\mathrm{GS}}$ holds. Therefore, if (4.3.2) is met, then by Theorem $3.3, z_{e}$ is stable in $T^{*} S E(3)$ modulo $\left(G_{a}\right)_{\mu_{a}}$ (S) $V$. We compute

$$
G_{a}=\{A \in S O(3) \mid A a=a\}=S^{1}
$$

and $\mu_{a} \in \mathfrak{g}_{a}^{*}=\mathfrak{s o}(2)^{*}$. Since $G_{a}$ is abelian, $\left(G_{a}\right)_{\mu_{a}}=G_{a}=S^{1}$. Thus, $z_{e}$ is stable in $T^{*} S E(3)$ modulo $S^{1}\left(\mathrm{~S} \mathbb{R}^{3}=S E(2) \times \mathbb{R}\right.$.

This is a stronger result than that given by the energy-Casimir method, since now the stability conclusion implies that the rotational parameters do not drift away from the equilibrium rotation axis. The vehicle may, however, experience drift in the translational parameters. The condition of Theorem 4.1 is also a necessary condition (leaving aside the case of equality) as follows from the results of Leonard [1995b].

We note that in his classical analysis of a submerged rigid body, Lamb [1932] does not comment on this kind of extended stability, i.e., he studies (linear) stability of equilibria of the momentum equations but does not address the issue of drift in the rotational and translational parameters.

The following example simulation illustrates that the extended stability result of Theorem 4.1, i.e., stability modulo $S E(2) \times \mathbb{R}$, is the best we can expect. That is, the translational parameters can indeed drift. Further, it is representative of simulation results for the equilibrium solutions studied in subsequent sections, indicating that the extended stability results for those cases too are the best we can expect. In this example, we choose an initial condition close to the equilibrium solution (4.3.1) and, using MATLAB, numerically compute the resulting phase space trajectory. Angular velocity $\Omega$ and linear velocity $v$ are computed by integrating (4.1.5). The attitude of the vehicle $R \in S O(3)$, which satisfies

$$
\dot{R}=R \hat{\Omega},
$$

is computed in terms of its local coordinate representation where local coordinates are chosen to be $\gamma_{1}, \gamma_{2}, \gamma_{3}$, (roll, pitch and yaw, respectively), defined through the relation

$$
R=e^{\gamma_{1} \hat{e}_{1}} e^{\gamma_{2} \hat{e}_{2}} e^{\gamma_{3} \hat{e}_{3}} .
$$

The position of the vehicle $b \in \mathbb{R}^{3}$ is the position of the origin of the body-fixed frame with respect to the inertial frame. $b$ is computed by integrating

$$
\dot{b}=R v .
$$

For our illustration we consider an ellipsoidal vehicle with semiaxis lengths $l_{1}=l_{2}=0.4 \mathrm{~m}$ and $l_{3}=0.75 \mathrm{~m}$ and with mass $m=500 \mathrm{~kg}$ so that the vehicle is (approximately) neutrally buoyant (density of water is $1000 \mathrm{~kg} / \mathrm{m}^{3}$ ). Accordingly, the inertia and mass matrix parameters are computed to be $I_{1}=I_{2}=87 \mathrm{~kg}-\mathrm{m}^{2}, I_{3}=32 \mathrm{~kg}-\mathrm{m}^{2}, m_{1}=m_{2}=840 \mathrm{~kg}, m_{3}=613 \mathrm{~kg}$. The 
equilibrium momentum values are $\Pi_{3}^{0}=I_{3} \Omega_{3}^{0}$ and $P_{3}^{0}=m_{3} v_{3}^{0}$ where we let equilibrium values of the velocities be $\Omega_{3}^{0}=10 \mathrm{rad} / \mathrm{s}$ and $v_{3}^{0}=1 \mathrm{~m} / \mathrm{s}$. Using Theorem 4.1, it is straightforward to check that this is a stable equilibrium modulo drift in $\gamma_{3}$ and $b$. Let the initial conditions be $\Omega(0)=(0.01,-0.008,10)^{T} \mathrm{rad} / \mathrm{s}, v(0)=(0.009,0.011,1)^{T} \mathrm{~m} / \mathrm{s}, \gamma(0)=0 \mathrm{rad}$ and $b(0)=0 \mathrm{~m}$. The trajectory of the vehicle for these initial conditions is given in Figures 4.3.1 and 4.3.2. Figure 4.3.1 shows plots of $\Omega$ and $v$ as a function of time. As expected there is no drift in velocity (and thus momentum). Figure 4.3.2 shows plots of attitude parameters $\gamma$ and position $b$ as a function of time. Note that the rotational parameters do not drift away from the equilibrium rotation axis. However, there is drift in the translational parameters, notably in $b_{1}$ and $b_{2}$.
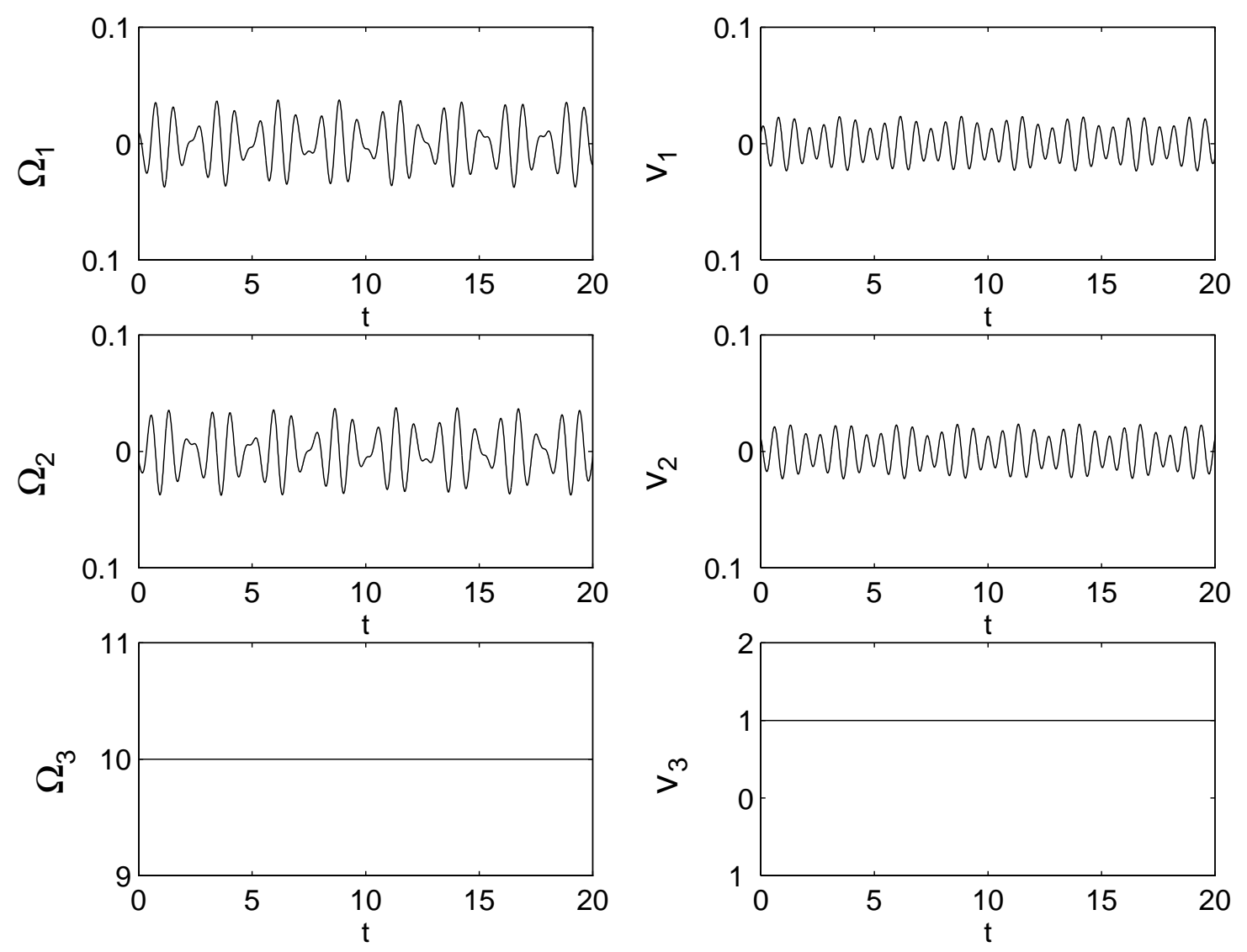

Figure 4.3.1: The angular velocity $(\mathrm{rad} / \mathrm{s})$ and translational velocity $(\mathrm{m} / \mathrm{s})$ versus time $(\mathrm{s})$ for the underwater vehicle of the simulation example.

\subsubsection{Generic Equilibria for a Vehicle with Noncoincident Centers}

The second case we examine is the vehicle with noncoincident centers and Lie-Poisson dynamics on $\mathfrak{w}^{*}$. Following the notation of $\S 3.3$, the phase space is $P_{\text {mech }}=T^{*} S E(3)=T^{*} \bar{G}=T^{*}(G$ (S $V)$, where $G=S O(3), V=\mathbb{R}^{3}$ and $\bar{G}=S E(3)$. The Poisson manifold $P$ is $P=T^{*} W$ where $W=S O(3) \subseteq\left(\mathbb{R}^{3} \times \mathbb{R}^{3}\right)=\left(S O(3)\left(\mathrm{S} \mathbb{R}^{3}\right)\left(\mathrm{S} \mathbb{R}^{3}\right.\right.$. The Poisson reduced space is $P / W=\mathfrak{w}^{*}=$ $\mathfrak{s o}(3)^{*} \times \mathbb{R}^{3^{*}} \times \mathbb{R}^{3^{*}}$, and the Poisson-reduced Hamiltonian is $h$ as given by (4.1.2). Let $a_{2} \in \mathbb{R}^{3^{*}}$, 

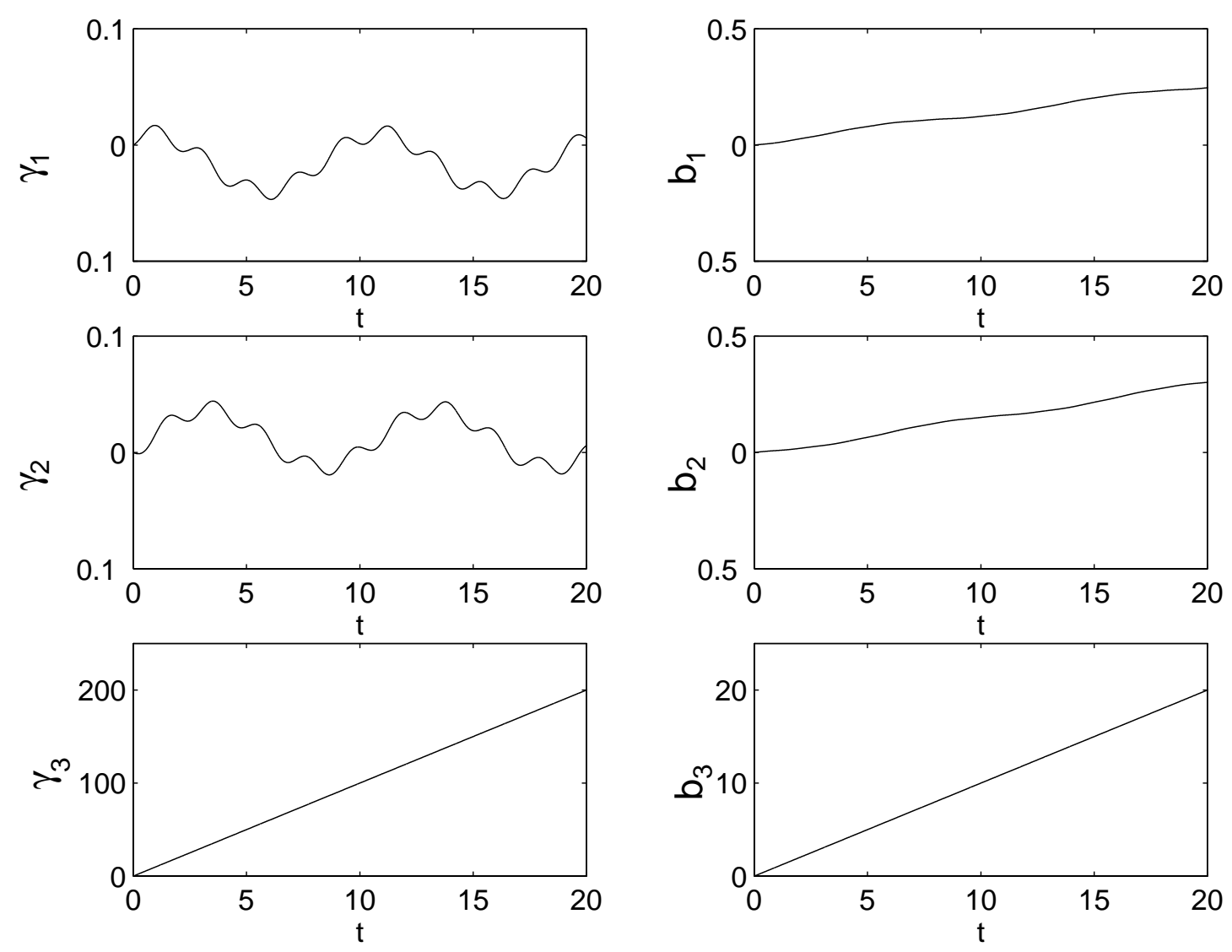

Figure 4.3.2: The attitude ( $\mathrm{rad}$ ) and position (m) versus time (s) for the underwater vehicle of the simulation example.

then $P_{\text {mech }}$ is the reduction of $P$ by $\mathbb{R}^{3}$ at $a_{2}$. Further, $\bar{G}_{a_{2}}=S E(2) \times \mathbb{R}$ is the symmetry group for the dynamics on $P_{\text {mech }}$, and the reduction of $P_{\text {mech }}$ by $\bar{G}_{a_{2}}$ at values $\left(\mu, a_{1}\right) \mid \overline{\mathfrak{g}}_{a_{2}} \in \mathfrak{s o}(3)^{*} \times \mathbb{R}^{3^{*}}$ is isomorphic to the coadjoint orbit through $\left(\mu, a_{1}, a_{2}\right) \in \mathfrak{w}^{*}$. Let $z_{\text {emech }} \in T^{*} S E(3)$ be a relative equilibrium for the action of $\bar{G}_{a_{2}}, z_{e}$ the corresponding equilibrium in $T^{*} W$ and $\left[\left[z_{e}\right]\right]=\left(\mu, a_{1}, a_{2}\right) \in$ $\mathfrak{w}^{*}$ the Poisson-reduced equilibrium. We let $a=\left(a_{1}, a_{2}\right) \in \mathbb{R}^{3 *} \times \mathbb{R}^{3^{*}}$.

Let the vehicle be ellipsoidal with inertia matrix $I=\operatorname{diag}\left(I_{1}, I_{2}, I_{3}\right)$ and mass matrix $M=$ $\operatorname{diag}\left(m_{1}, m_{2}, m_{3}\right)$. Further, assume that $r_{G}=(0,0, l)^{T}$, i.e., the center of gravity is located along the third principal axis a distance $|l|$ from the center of buoyancy (recall Figure 1.3.1). Consider the one-parameter family of equilibrium solutions

$$
\mu=\left(-m l P_{2}^{0} / m_{2}, 0,0\right)^{T}, \quad a_{1}=\left(0, P_{2}^{0}, 0\right)^{T}, \quad a_{2}=(0,0,1)^{T} .
$$

This corresponds to the body oriented so that the third principal axis is parallel to gravity with steady translation (but no spin) along one of the other principal axes (perpendicular to gravity). As long as $P_{2}^{0} \neq 0$, this relative equilibrium is generic. In Leonard [1995b], the author showed for such a generic equilibrium that Assumption 2DSDP is satisfied if and only if

$$
l>0, \quad m_{2}>m_{1}, \quad m g l>\left(\frac{1}{m_{2}}-\frac{1}{m_{3}}\right)\left(P_{2}^{0}\right)^{2} .
$$


The condition $l>0$ requires that the vehicle be bottom heavy, i.e., with center of gravity lower than center of buoyancy at the equilibrium. Recall that $m_{i}$ is inversely related to the length of the ellipsoid's $i$ th semiaxis $l_{i}$. Thus, if the axis of translation $l_{2}$ is the shortest of three axes, then $m_{2}>m_{1}$ and $m_{2}>m_{3}$, and conditions (4.3.4) are met as long as $l>0$. If the axis of translation $l_{2}$ is the intermediate axis and the axis parallel to gravity is the shortest axis, i.e., $l_{1}>l_{2}>l_{3}$, then $m_{3}>m_{2}>m_{1}$, and conditions (4.3.4) are met provided that $l$ is positive and sufficiently large for a given translation. If the axis of translation $l_{2}$ is the longest axis, then conditions (4.3.4) are never met. By the energy-Casimir method, if (4.3.4) is met, then $z_{e \text { emech }}$ is stable in $T^{*} S E(3)$ modulo $S E(2) \times \mathbb{R}$.

Using the extended stability theorem 3.5, we can say more about the stability of $z_{\text {emech }}$.

Theorem 4.2 If (4.3.4) is met, then $z_{e m e c h}$ as described by (4.3.3) is stable in $T^{*} S E(3)$ modulo $\mathbb{R}^{3}$.

Proof Since $G=S O(3)$ is compact, Assumption $1_{\mathrm{DSDP}}$ holds. Therefore, if (4.3.4) is met, then by Theorem $3.5, z_{e \text { emech }}$ is stable in $T^{*} S E(3)$ modulo $\left(G_{a}\right)_{\mu_{a}}$ S $V$. We compute

$$
G_{a}=\left\{A \in S O(3) \mid A a_{1}=a_{1}, A a_{2}=A a_{2}\right\}=e,
$$

$e$ the identity in $S O(3)$. Thus, $\left(G_{a}\right)_{\mu_{a}}=e$ and so $z_{e \text { mech }}$ is stable in $T^{*} S E(3)$ modulo $\mathbb{R}^{3}$.

This is a stronger result than that given by the energy-Casimir method, since now the stability conclusion implies that there is no drift in rotational parameters.

\subsection{Stability of Nongeneric Momentum Values}

In this section we apply the stability theorems of $\S 3.4$ to nongeneric equilibria of the underwater vehicle. Nongeneric equilibria of the underwater vehicle were discussed in Leonard [1995b], and conditions for instability were determined using linearization. However, conditions for nonlinear stability could not be fully determined using the energy-Casimir method alone. Here we make use of subcasimirs and the additional sufficient conditions derived in $\S 3.4$ to prove conditions for nonlinear stability of the nongeneric equilibria.

As was the case in $\S 4.3$, our extension of Patrick's result using reduction by stages is essential. This again is a result of the fact that for our examples, the symmetry group is not compact.

\subsubsection{Nongeneric Equilibria for a Vehicle with Coincident Centers}

The first case we examine is the vehicle with coincident centers of buoyancy and gravity and Lie-Poisson dynamics on $\mathfrak{s e}(3)^{*}$. The setup is the same as in $\S 4.3 .1$ except that we consider the one-parameter family of nongeneric equilibrium solutions

$$
\mu=\left(0,0, \Pi_{3}^{0}\right)^{T}, \quad a=(0,0,0)^{T}
$$

which corresponds to steady rotation about one of the principal axes of the vehicle (and no translation). Further, to begin we do not assume that the axis of rotation is an axis of symmetry.

Recall from $\S 4.1$ that the orbit through this equilibrium is two-dimensional and there are two Casimirs, $\Pi \cdot \mathrm{P}$ and $\|\mathrm{P}\|^{2}$, and one subcasimir $\|\Pi\|^{2}$, where $(\Pi, \mathrm{P}) \in \mathfrak{s e}(3)^{*}$. To satisfy Assumption 
$2_{\mathrm{GS}}$, we show that there exists a function $\Phi: \mathbb{R}^{3} \rightarrow \mathbb{R}$ such that

$$
h_{\Phi}=\frac{1}{2}\left(\Pi^{T} A \Pi+\mathrm{P}^{T} C \mathrm{P}\right)+\Phi\left(\Pi \cdot \mathrm{P},\|\mathrm{P}\|^{2},\|\Pi\|^{2}\right)
$$

has a critical point at $(\Pi, \mathrm{P})=(\mu, a)$ and such that the second variation of $h_{\Phi}$ evaluated at $(\mu, a)$ is definite.

To do this, define

$$
\dot{\Phi}=\frac{\partial \Phi}{\partial(\Pi \cdot \mathrm{P})}, \quad \Phi^{\prime}=\frac{\partial \Phi}{\partial\left(\|\mathrm{P}\|^{2}\right)}, \quad \Phi^{\dagger}=\frac{\partial \Phi}{\partial\left(\|\Pi\|^{2}\right)} .
$$

When evaluated at the equilibrium the first derivative of $h_{\Phi}$ is zero if and only if at the equilibrium

$$
\dot{\Phi}=0, \quad 2 \Phi^{\dagger}=-\frac{1}{I_{3}} .
$$

$\Phi^{\prime}$ can be arbitrary at the equilibrium.

The matrix of the second derivative of $h_{\Phi}$ at the equilibrium, where we make the substitutions from $(4.4 .2)$, is

$$
\left(\begin{array}{cccccc}
\frac{1}{I_{1}}-\frac{1}{I_{3}} & 0 & 0 & 0 & 0 & 0 \\
0 & \frac{1}{I_{2}}-\frac{1}{I_{3}} & 0 & 0 & 0 & 0 \\
0 & 0 & \left(\Pi_{3}^{0}\right)^{2} \Phi^{\dagger \dagger} & 0 & 0 & 2\left(\Pi_{3}^{0}\right)^{2} \dot{\Phi}^{\dagger} \\
0 & 0 & 0 & \frac{1}{m_{1}}+2 \Phi^{\prime} & 0 & 0 \\
0 & 0 & 0 & 0 & \frac{1}{m_{2}}+2 \Phi^{\prime} & 0 \\
0 & 0 & 2\left(\Pi_{3}^{0}\right)^{2} \dot{\Phi}^{\dagger} & 0 & 0 & \frac{1}{m_{3}}+2 \Phi^{\prime}+\left(\Pi_{3}^{0}\right)^{2} \ddot{\Phi}
\end{array}\right)
$$

where first and second partials of $\Phi$ are evaluated at $(\mu, a)$. For positive definiteness of this matrix, it is clearly necessary that

$$
\frac{1}{I_{1}}-\frac{1}{I_{3}}>0, \quad \frac{1}{I_{2}}-\frac{1}{I_{3}}>0
$$

It can also be seen to be sufficient if we choose the equilibrium values of the partials of $\Phi$ such that $\Phi^{\dagger \dagger}>0$ and $\Phi^{\prime}=\dot{\Phi}^{\dagger}=\ddot{\Phi}=0$. Similarly, for negative definiteness, it is necessary that

$$
\frac{1}{I_{1}}-\frac{1}{I_{3}}<0, \quad \frac{1}{I_{2}}-\frac{1}{I_{3}}<0
$$

It is seen to be sufficient by choosing the equilibrium values of the partials of $\Phi$ such that $\Phi^{\dagger \dagger}<0$, $2 \Phi^{\prime}<\min _{i=1,2,3}\left(-1 / m_{i}\right)$ and $\dot{\Phi}^{\dagger}=\ddot{\Phi}=0$. Thus, the second variation of $h_{\Phi}$ at $(\mu, a)$ is definite if and only if

$$
\left\{I_{3}>I_{1}, \quad \text { and } \quad I_{3}>I_{2}\right\} \quad \text { or } \quad\left\{I_{3}<I_{1}, \quad \text { and } \quad I_{3}<I_{2}\right\} \text {. }
$$

This condition is sufficient for leafwise stability of the pure rotation. Using linearization, one can show that the condition is also a necessary condition for stability (leaving aside the case of equality). The preceding condition is the same as that for stability of a free rigid body in space; 
however, the implications for the submerged rigid body are different in the following way. In both cases the rotation is stable (leafwise for the submerged body) if the moment of inertia about the axis of rotation is either the largest or the smallest of the three moments of inertia. For the free rigid body in space, this corresponds to stability about the longest and shortest axes and instability about the intermediate axis. For the submerged rigid body, because the moments of inertia include added inertia terms due to the presence of the fluid, the intermediate moment of inertia of the bodyfluid system can correspond to the long, short or the intermediate length axis. That is, depending upon the configuration of the body, the unstable axis can be the long, short or intermediate axis. See Leonard [1995b] for background on added inertia.

Proving stability of the pure rotation in the full phase space appears to be much more delicate (even than the nongeneric case of the rising bottom-heavy vehicle of $\S 4.4 .2$ ). One can show that the space $F$ has dimension greater than one and so we cannot use Theorem 3.6 or 3.7. One could possibly make use of an argument like that given in Remark 1 following Theorem 3.6. Rather than pursue this here we show in the following the stability result when we assume that the vehicle is symmetric about the axis of rotation, i.e., $I_{1}=I_{2}$ and $m_{1}=m_{2}$.

In this case with the additional symmetry, $\Pi_{3}$ is a conserved quantity. To satisfy Assumption $2_{\mathrm{GS}}$, we consider the conserved function

$$
h_{\Phi}^{\prime}=\frac{1}{2}\left(\Pi^{T} A \Pi+\mathrm{P}^{T} C \mathrm{P}\right)+\Phi\left(\Pi \cdot \mathrm{P},\|\mathrm{P}\|^{2}\right)+\phi\left(\Pi_{3}\right),
$$

where $\Phi: \mathbb{R}^{2} \rightarrow \mathbb{R}$ and $\phi: \mathbb{R} \rightarrow \mathbb{R}$. Note that this function does not include subcasimirs.

A simple computation shows that the first derivative of $h_{\Phi}^{\prime}$ is zero at the equilibrium if and only if $\phi^{\prime}=-\Pi_{3}^{0} / I_{3}$ and $\Phi^{\prime}=0$ when evaluated at the equilibrium. Further, choosing the remaining partial derivatives of $\phi$ and $\Phi$ to be zero yields a (positive) definite second derivative of $h_{\Phi}^{\prime}$ at the equilibrium. Thus, Assumption $2_{\mathrm{GS}}$ is satisfied. Assumption $3_{\mathrm{NG}}$ is also satisfied since $h_{\Phi}^{\prime}$ is a dynamically invariant function (see Remark 2 following Assumption $3_{\mathrm{NG}}$ ).

Theorem 4.3 Assuming that the axis of rotation is an axis of symmetry, $z_{e}$ as defined by (4.4.1) is stable in $T^{*} S E(3)$ modulo $S E(2) \times \mathbb{R}$.

Proof Since $G=S O(3)$ is compact, Assumption $1_{\mathrm{GS}}$ holds. Since Assumptions $2_{\mathrm{GS}}$ and $3_{\mathrm{NG}}$ hold, by Theorem 3.6, $z_{e}$ is stable in $T^{*} S E(3)$ modulo $\left(G_{a}\right)_{\mu_{a}}$ (S) $V$. We compute

$$
G_{a}=\{A \in S O(3) \mid A a=a\}=S O(3) .
$$

Thus, $\mathfrak{g}_{a}=\mathfrak{s o}(3)$, so $\mu_{a} \in \mathfrak{g}_{a}^{*}=\mu$. We compute

$$
\left(G_{a}\right)_{\mu_{a}}=\{A \in S O(3) \mid A \mu=\mu\}=S^{1} .
$$

Thus, $z_{e}$ is stable in $T^{*} S E(3)$ modulo $S^{1}\left(\mathbb{R}^{3}=S E(2) \times \mathbb{R}\right.$.

\subsubsection{Nongeneric Equilibria for a Vehicle with Noncoincident Centers}

The second case we examine is the vehicle with centers of buoyancy and gravity that are noncoincident and Lie-Poisson dynamics on $\mathfrak{w}^{*}$. The setup is the same as in $\S 4.3 .2$ except that we consider the two-parameter family of nongeneric equilibrium solutions

$$
\mu=\left(0,0, \Pi_{3}^{0}\right)^{T}, \quad a_{1}=\left(0,0, P_{3}^{0}\right)^{T}, \quad a_{2}=(0,0,1)^{T} .
$$


This corresponds to the body oriented so that the third principal axis is parallel to gravity with the body rotating about and translating along this same axis, i.e., the body rises or falls and spins about the axis of gravity. Further, suppose that the vehicle is symmetric about the axis of rotation, i.e., $I_{1}=I_{2}$ and $m_{1}=m_{2}$. Then $\Pi_{3}$ is a conserved quantity.

Recall from $\S 4.1$ that the orbit through this equilibrium is four-dimensional and there are three Casimirs, $\mathrm{P} \cdot \Gamma,\|\mathrm{P}\|^{2}$ and $\|\Gamma\|^{2}$, and two subcasimirs $\Pi \cdot \mathrm{P}$ and $\Pi \cdot \Gamma$, where $(\Pi, \mathrm{P}, \Gamma) \in \mathfrak{w}^{*}$. To satisfy Assumption $2_{\mathrm{DSDP}}$, we need to show that there exist functions $\Phi: \mathbb{R}^{5} \rightarrow \mathbb{R}$ and $\phi: \mathbb{R} \rightarrow \mathbb{R}$ such that

$$
h_{\Phi}=\frac{1}{2}\left(\Pi^{T} A \Pi+2 \Pi^{T} B^{T} \mathrm{P}+\mathrm{P}^{T} C \mathrm{P}-2 m g l\left(\Gamma \cdot e_{3}\right)\right)+\Phi\left(\mathrm{P} \cdot \Gamma,\|\mathrm{P}\|^{2},\|\Gamma\|^{2}, \Pi \cdot \mathrm{P}, \Pi \cdot \Gamma\right)+\phi\left(\Pi_{3}\right)
$$

has a critical point at $(\Pi, \mathrm{P}, \Gamma)=\left(\mu, a_{1}, a_{2}\right)$ and such that the second variation of $h_{\Phi}$ evaluated at $\left(\mu, a_{1}, a_{2}\right)$ is definite.

Define

$$
\dot{\Phi}=\frac{\partial \Phi}{\partial(\mathrm{P} \cdot \Gamma)}, \quad \Phi^{\prime}=\frac{\partial \Phi}{\partial\left(\|\mathrm{P}\|^{2}\right)}, \quad \Phi^{\dagger}=\frac{\partial \Phi}{\partial\left(\|\Gamma\|^{2}\right)}, \quad \Phi^{a}=\frac{\partial \Phi}{\partial(\Pi \cdot \mathrm{P})}, \quad \Phi^{b}=\frac{\partial \Phi}{\partial(\Pi \cdot \Gamma)} .
$$

When evaluated at the equilibrium the first derivative of $h_{\Phi}$ is zero if and only if at the equilibrium

$$
\begin{aligned}
\phi^{\prime} & =-\frac{\Pi_{3}^{0}}{I_{3}}+\Phi^{a} P_{3}^{0}+\Phi^{b} \\
\dot{\Phi} & =-\left(\frac{1}{m_{3}}+2 \Phi^{\prime}\right) P_{3}^{0}-\Phi^{a} \Pi_{3}^{0} \\
2 \Phi^{\dagger} & =m g l-\Phi^{b} \Pi_{3}^{0}+\left(\frac{1}{m_{3}}+2 \Phi^{\prime}\right)\left(P_{3}^{0}\right)^{2}+\Phi^{a} \Pi_{3}^{0} P_{3}^{0} .
\end{aligned}
$$

The equilibrium values of $\Phi^{\prime}, \Phi^{a}$ and $\Phi^{b}$ can be arbitrary.

A long calculation (done with the help of Mathematica) shows that with the appropriate choices of the second partials and the unrestricted first partials of $\Phi$, the matrix of the second derivative of $h_{\Phi}$ at the equilibrium is (positive) definite (i.e., Assumption $22_{\mathrm{DSDP}}$ holds) if and only if

$$
m g l>\left(\frac{1}{m_{3}}-\frac{1}{m_{2}}\right)\left(P_{3}^{0}\right)^{2}-\left(\frac{1}{\bar{a}_{2}}\left(\Phi^{a}\right)^{2}\left(P_{3}^{0}\right)^{2}+\Phi^{a} \Pi_{3}^{0} P_{3}^{0}\right)
$$

where

$$
\bar{a}_{2}=\frac{m_{1}}{m_{1} I_{2}-m^{2} l^{2}} .
$$

To minimize the right hand side of (4.4.5), choose

$$
\Phi^{a}=-\frac{\bar{a}_{2} \Pi_{3}^{0}}{2 P_{3}^{0}}
$$

Then, (4.4.5) becomes

$$
m g l>\left(\frac{1}{m_{3}}-\frac{1}{m_{2}}\right)\left(P_{3}^{0}\right)^{2}-\frac{\bar{a}_{2}}{4}\left(\Pi_{3}^{0}\right)^{2}
$$

Theorem 4.4 If (4.4.6) is met, then $z_{\text {emech }}$ as defined by (4.4.4) is stable in $T^{*} S E(3)$ modulo $S E(2) \times \mathbb{R}$. 
Proof Since $G=S O(3)$ is compact, Assumption 1DSDP holds. Therefore, if (4.4.6) is met and

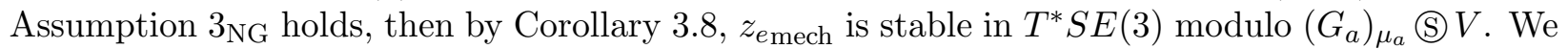
compute

$$
G_{a}=\left\{A \in S O(3) \mid A a_{1}=a_{1}, A a_{2}=a_{2}\right\}=S^{1} .
$$

and $\mu_{a} \in \mathfrak{g}_{a}^{*}=\mathfrak{s o}(2)^{*}$. Since $G_{a}$ is abelian, $\left(G_{a}\right)_{\mu_{a}}=G_{a}=S^{1}$.

To complete the proof we check Assumption $3_{\mathrm{NG}}$. First of all, we compute the space $F$. The coadjoint action of $W$ on $\mathfrak{w}^{*}$ has the expression

$$
(R, b, w)(x, y, z)=(R x+b \times R y+w \times R z, R y, R z) .
$$

In this case, the space $E$ corresponds to the noncompact orbit through the nongeneric point $\left(\mu, a_{1}, a_{2}\right)=\left(\mu, a_{1}, P_{3}^{0} a_{1}\right)$, namely the space $E$ of vectors of the form $\left(\left(b+P_{3}^{0} w\right) \times a_{1}, 0,0\right)$ for arbitrary vectors $b$ and $w$. This space is two dimensional. The corresponding space for nearby generic points $\left(\mu^{\prime}, a_{1}^{\prime}, a_{2}^{\prime}\right), a_{1}^{\prime} \nVdash a_{2}^{\prime}$, is $E^{\prime}$, the space of vectors of the form $\left(b \times a_{1}^{\prime}+w \times a_{2}^{\prime}, 0,0\right)$, which is three dimensional. Thus, we can choose as $F$, the space of vectors of the form $\left(a_{1}, 0,0\right)$. Next, we choose the function $h+\phi$ where $\phi\left(\Pi_{3}\right)$ satisfies $\phi^{\prime}\left(\Pi_{3}^{0}\right)=-\Pi_{3}^{0} / I_{3}$ and $\phi^{\prime \prime}\left(\Pi_{3}^{0}\right)>-1 / I_{3}$. This satisfies Assumption $33_{\mathrm{NG}}$ since at the equilibrium it has a critical point in the direction of $F$ and its second derivative in that direction is $\left(1 / I_{3}\right)+\phi^{\prime \prime}\left(\Pi_{3}^{0}\right)$ which is positive. Thus, $z_{e m e c h}$ is stable in $T^{*} S E(3)$ modulo $S^{1}\left(\mathbb{R}^{3}=S E(2) \times \mathbb{R}\right.$.

The condition of Theorem 4.4 is also a necessary condition (leaving aside the case of equality), as follows from the results of Leonard [1995b].

Remark. In the stability analysis of the heavy top (see Lewis et al [1992]) one can view the top as a system on $T^{*} \mathrm{SO}(3)$ with $S^{1}$ symmetry (rotations about the axis of gravity) and by the semidirect product theory, the reduction leads to a Lie-Poisson system on $\mathfrak{s e}(3)^{*}$. When the top has an additional body axis of symmetry, one gets an additional conserved quantity that is used in the stability analysis on $\mathfrak{s e}(3)^{*}$. However, for the upright top, care is needed since the momentum of this state for the whole symmetry group $S^{1} \times S^{1}$ including the body symmetry, is not a regular value. A similar caution is needed in the underwater vehicle when it has an axis of symmetry. However, we do not include this extra symmetry in our basic set up and only use it as an aid in the stability analysis in the Lie-Poisson setting, so this point does not cause problems.

The condition (4.4.6) says that both spin and low center of gravity serve to stabilize the equilibrium motion. The motion can be stabilized, whether or not the axis of rotation and translation is the short axis or the long axis, as long as the center of gravity is sufficiently low and/or the angular velocity is sufficiently high. It is easier to stabilize the case in which the axis of motion is the short axis rather than the long axis. However, the motion of a top-heavy body can be stabilized with fast enough spin even when the axis of motion is the long axis.

We showed the last step of the analysis of positive definiteness to make note of the fact that only with the use of the subcasimirs is it possible to derive the condition (4.4.6). Recall that $\Phi^{a}$ is the partial derivative of $\Phi$ with respect to the subcasimir $\Pi \cdot \mathrm{P}$. If we had analyzed this case without this subcasimir, then $\Phi^{a}$ would have been identically zero and the last term in (4.4.6) would be missing, i.e., the stabilizing effect of spin would not have been evident. 
In other words, to prove stability under the more restrictive condition that

$$
m g l>\left(\frac{1}{m_{3}}-\frac{1}{m_{2}}\right)\left(P_{3}^{0}\right)^{2},
$$

we could use the simpler conserved function

$$
h_{\Phi}^{\prime}=\frac{1}{2}\left(\Pi^{T} A \Pi+2 \Pi^{T} B \mathrm{P}+\mathrm{P}^{T} C \mathrm{P}-2 m g l\left(\Gamma \cdot e_{3}\right)\right)+\Phi\left(\mathrm{P} \cdot \Gamma,\|\mathrm{P}\|^{2},\left\|\Gamma^{2}\right\|\right)+\phi\left(\Pi_{3}\right),
$$

which does not include the subcasimirs. At the nongeneric equilibrium, this function has a critical point as a function on all of $\mathfrak{w}^{*}$ and the second derivative is positive definite if condition (4.4.7) holds. Therefore, Assumption $33_{\mathrm{NG}}$ follows trivially, as discussed in the remark that precedes Theorem 3.6, and stability follows. The transition from condition (4.4.7) to condition (4.4.6) corresponds to a passing of imaginary eigenvalues of the linearization of the dynamics at the equilibrium point as discussed below.

This case reveals some interesting bifurcation phenomena including a Hamiltonian Hopf bifurcation and the passing of eigenvalues on the imaginary axis. To examine these, we look at the eigenvalues of the linearization of the dynamics (4.1.3) at the equilibrium (4.4.4) as the equilibrium linear momentum $P_{3}^{0}$ is varied. The characteristic polynomial for the linearization at the equilibrium is

$$
\lambda^{3}\left(\lambda^{2}+\left(\frac{\Pi_{3}^{0}}{I_{3}}\right)^{2}\right)\left(\lambda^{4}+p \lambda^{2}+q\right)
$$

where

$$
\begin{gathered}
p=2 \bar{a}_{2}\left(\frac{1}{m_{2}}-\frac{1}{m_{3}}+\frac{m g l}{\left(P_{3}^{0}\right)^{2}}\right)\left(P_{3}^{0}\right)^{2}+\left(1+\left(1-\bar{a}_{2} I_{3}\right)^{2}\right)\left(\frac{\Pi_{3}^{0}}{I_{3}}\right)^{2}, \\
q=\left(\bar{a}_{2}\left(\frac{1}{m_{2}}-\frac{1}{m_{3}}+\frac{m g l}{\left(P_{3}^{0}\right)^{2}}\right)\left(P_{3}^{0}\right)^{2}-\left(1-\bar{a}_{2} I_{3}\right)\left(\frac{\Pi_{3}^{0}}{I_{3}}\right)^{2}\right)^{2} .
\end{gathered}
$$

There are three eigenvalues fixed at the origin and two eigenvalues fixed at $\pm\left(\Pi_{3}^{0} / I_{3}\right) i$. The remaining four eigenvalues, the roots of the quartic polynomial factor, move as the parameter $P_{3}^{0}$ is varied. These eigenvalues are on the imaginary axis as long as condition (4.4.6) is met. Suppose $m_{3}<m_{2}$ (i.e., $l_{3}>l_{2}$ ) and $m g l>0$. As $P_{3}^{0}$ is increased, the pair of eigenvalues above the real axis and the pair below each meet and then split off the imaginary axis. The point at which each pair of eigenvalues meets, i.e., the Hamiltonian Hopf bifurcation point, corresponds to the value of $P_{3}^{0}$ that makes condition (4.4.6) an equality.

Before the Hamiltonian Hopf bifurcation occurs, i.e., while the eigenvalues are all on the imaginary axis, each of the eigenvalues fixed at $\pm\left(\Pi_{3}^{0} / I_{3}\right) i$ is passed by one of the moving eigenvalues. This passing (resonance) of eigenvalues occurs when $P_{3}^{0}$ is such that condition (4.4.7) holds with equality, i.e.,

$$
m g l=\left(\frac{1}{m_{3}}-\frac{1}{m_{2}}\right)\left(P_{3}^{0}\right)^{2},
$$

at which point the quartic polynomial becomes

$$
\lambda^{4}+p \lambda^{2}+q=\left(\lambda^{2}+\left(\frac{\Pi_{3}^{0}}{I_{3}}\right)^{2}\right)\left(\lambda^{2}+\left(1-\bar{a}_{2} I_{3}\right)^{2}\left(\frac{\Pi_{3}^{0}}{I_{3}}\right)^{2}\right)
$$


We note that the second derivative of the augmented Hamiltonian $h_{\Phi}$ at the equilibrium is positive definite throughout this passing of eigenvalues, while the second derivative of $h_{\Phi}^{\prime}$ loses definiteness at this passing. For a generic equilibrium one expects a change in definiteness for an eigenvalue passing and further that symmetry breaking will destroy stability. Here, because we are at a nongeneric point and there is a conserved quantity that does not lose definiteness, it is possible that symmetry breaking might not destroy stability. See van der Meer [1985, 1990] and Golubitsky, Marsden, Stewart, and Dellnitz [1995] for discussions of the Hamiltonian Hopf bifurcation and Dellnitz, Melbourne and Marsden [1992] for a discussion of eigenvalue movement for the Hamiltonian Hopf bifurcation for Hamiltonian systems with symmetry. Stability analysis of the rising and spinning, bottom-heavy underwater vehicle without an axis of symmetry will make use of Assumption $3_{\mathrm{NG}}^{\prime}$, since without the conserved quantity $\phi\left(\Pi_{3}\right)$, it may not be possible to prove Assumption $3_{\mathrm{NG}}$. Further investigation of bifurcation phenomena is left for future work.

The plot of Figure 4.4.1, generated by MATLAB, illustrates the movement of the eigenvalues and the bifurcation and eigenvalue passing phenomena. For this illustration we let inertia and mass matrix parameters be $I_{1}=I_{2}=87 \mathrm{~kg}-\mathrm{m}^{2}, I_{3}=32 \mathrm{~kg}-\mathrm{m}^{2}, m_{1}=m_{2}=840 \mathrm{~kg}, m_{3}=613 \mathrm{~kg}$ and $m=500 \mathrm{~kg}, g=9.8 \mathrm{~m} / \mathrm{s}^{2}, l=0.1 \mathrm{~m}$. The equilibrium angular momentum is $\Pi_{3}^{0}=150 \mathrm{~kg}-\mathrm{m}^{2} / \mathrm{s}$ and $P_{3}^{0}$ is increased from 400 to $1400 \mathrm{~kg}-\mathrm{m} / \mathrm{s}$. The five eigenvalues that remain fixed are drawn as circles (note there are three eigenvalues at the origin). The crosses indicate the positions of the four remaining eigenvalues at the point when $P_{3}^{0}=400 \mathrm{~kg}-\mathrm{m} / \mathrm{s}$. The dotted lines show the paths of these four eigenvalues as $P_{3}^{0}$ is increased to 1400 . The eigenvalue crossing occurs when $P_{3}^{0} \approx 1054$ $\mathrm{kg}-\mathrm{m} / \mathrm{s}$, and the Hamiltonian Hopf bifurcation point corresponds to $P_{3}^{0} \approx 1124 \mathrm{~kg}-\mathrm{m} / \mathrm{s}$.

\section{Future Directions}

As was described in Leonard [1995b] and references therein, one of the main goals of future work in this area is to make use of various control strategies to control the movement of underwater vehicles. The work of Leonard [1995a,c] and Leonard and Krishnaprasad [1995] has already shown that control strategies using Lie group methods are useful for attitude and position control. We believe that the setup of the problem as we have described it will be useful for other endeavors along these lines. For example, the setup has already been used to derive feedback controls for stabilizing otherwise unstable equilibria in Leonard [1996]. The technique is related to the work of Bloch, Krishnaprasad, Marsden and Sánchez [1992] (see also Bloch, Marsden and Sánchez [1996]). On the other hand, the techniques of saddle point control (see Bloch and Marsden [1989], Coller [1995] and references therein) may be useful in open-loop control problems where one has limited actuation energy. Future work in investigating bifurcation phenomena will complement this effort.

Another direction that warrants further investigation is making more realistic models of the fluid dynamics, especially in cases when vorticity is generated by the body-fluid interaction. Normal form theory as in Langford and Zhan [1994] should be helpful in this regard. Including elastic and flexible properties of the body would also be interesting.

Another item that requires additional attention in this situation is the effect of dissipation. For example, the results of Haller [1992], Bloch, Krishnaprasad, Marsden and Ratiu [1994, 1996] (and references therein) would be interesting to study in the present context. Specifically, that theory says that at relative equilibria where the second variation is indefinite but the eigenvalues are on the imaginary axis, one gets a linear instability when small dissipation is added. This dissipation can be of Rayleigh dissipation function type when viewed from the point of view of the dynamics on 


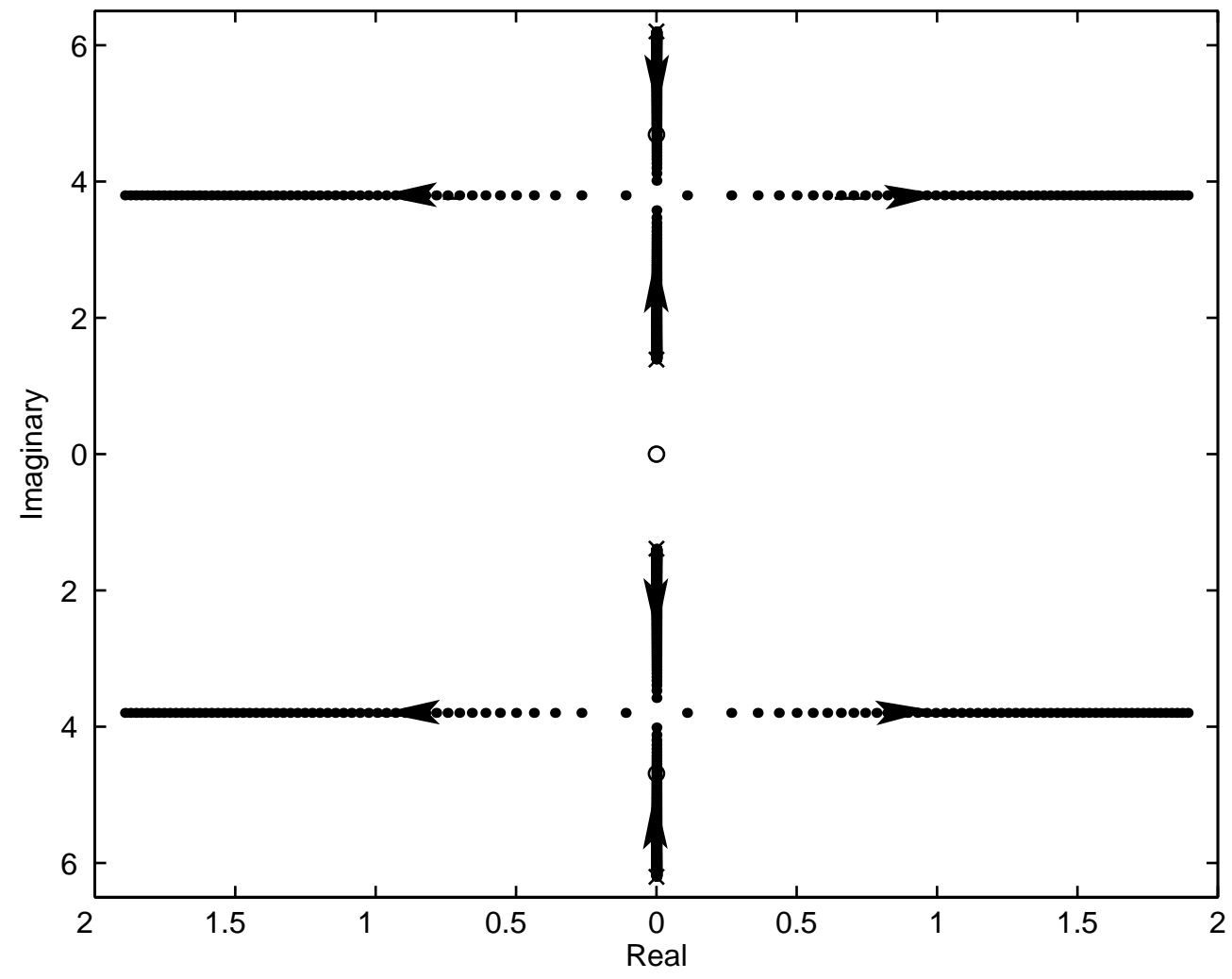

Figure 4.4.1: Hamiltonian Hopf bifurcation and eigenvalue passing for eigenvalues of linearization as $P_{3}^{0}$ is varied from 400 to $1400 \mathrm{~kg}-\mathrm{m} / \mathrm{s}$. The eigenvalues that remain fixed are identified by circles. The crosses identify the four other eigenvalues when $P_{3}^{0}=400 \mathrm{~kg}-\mathrm{m} / \mathrm{s}$. The dotted lines and arrows show the paths of these four eigenvalues as $P_{3}^{0}$ is increased to $1400 \mathrm{~kg}-\mathrm{m} / \mathrm{s}$.

the physical configuration space, namely $T^{*} S E(3)$, or can be of Brockett double bracket type when viewed from the Lie-Poisson point of view. Obviously, the effects of the addition of dissipation is important in the underwater vehicle problem; since the dissipation is often small, the present point of view should be useful.

The dynamic bifurcations that are observed in our analysis, especially the Hamiltonian Hopf bifurcation, need to be studied in more detail. In addition, the effects of symmetry breaking (such as the $S^{1}$ symmetry of the vehicle in the case of the rising vehicle) needs additional attention. The techniques of Knobloch, Mahalov and Marsden [1994] may prove useful in this regard. A complication in this regard is that, because of the nongeneric nature of the coadjoint orbit, the theory of eigenvalue movement (see Dellnitz, Melbourne and Marsden [1992]) requires additional work. All of these aspects of the underwater vehicle problem should provide interesting additional motivations for the continued development of the basic theory.

\section{Conclusions}

In this paper we have used the method of reduction by stages to generalize the stability results of Patrick, which allow nongeneric momentum values, to include the case in which the appropriate 
groups do not satisfy the required properness conditions. We have related the method to the energy-Casimir technique as an aid to check the hypotheses.

We have applied this stability theory to underwater vehicle dynamics, treating both the cases of relative equilibria whose momenta are generic as well as those that are not generic. We have found explicit criteria for stability and have observed phase drifts consistent with the theory. Moreover, for a rising, bottom-heavy vehicle, as the vertical momentum (impulse) is increased, a Hamiltonian Hopf bifurcation is identified.

\section{Appendix: Reduction by Stages}

This appendix is devoted to the proof of the reduction by stages theorem (3.1). We shall first check that the group $G_{a}$ acts symplectically on the reduced space $P_{a}$ and that it has a momentum map $\mathbf{J}_{a}$. As we shall see, the momentum map will be induced by a natural construction.

To see this, first note that the group $G_{a}$ leaves the set $\mathbf{J}_{V}^{-1}(a)$ invariant. Indeed, suppose that $\mathbf{J}_{V}(z)=a$ and that $g \in G$ leaves $a$ invariant. By equivariance, we have $\mathbf{J}_{V}(g z)=g \mathbf{J}_{V}(z)=g a=a$. Thus, $G_{a}$ acts on the set $\mathbf{J}_{V}^{-1}(a)$. We denote this action by $\Psi_{g}^{a}: \mathbf{J}_{V}^{-1}(a) \rightarrow \mathbf{J}_{V}^{-1}(a)$.

The action $\Psi^{a}$ induces an action $\Psi_{a}$ on the quotient space $\mathbf{J}_{V}^{-1}(a) / V$ as follows. If we let elements of the quotient space $\mathbf{J}_{V}^{-1}(a) / V$ be denoted by $[z]$, regarded as equivalence classes, then we claim that $g[z]=[g z]$ defines the induced action on the quotient space. We only need to show that it is well defined; indeed, suppose that $v \in V$ so that $[z]=[v z]$. Identifying $v=(e, v)$ and $g=(g, 0)$ in the semidirect product, we have,

$$
[g v z]=[(g, 0)(e, v) z]=[(e, g v)(g, 0) z]=[(g v)(g z)]=[g z] .
$$

Thus, the action $\Psi_{a}$ of $G_{a}$ on the $V$-reduced space $P_{a}$ is well defined. The action of a group element $g \in G_{a}$ will be denoted by $\Psi_{g, a}: P_{a} \rightarrow P_{a}$. We shall next show that this action is symplectic.

Let $\pi_{a}: \mathbf{J}_{V}^{-1}(a) \rightarrow P_{a}$ denote the natural projection and $i_{a}: \mathbf{J}_{V}^{-1}(a) \rightarrow P$ be the inclusion. By construction, $\Psi_{g, a} \circ \pi_{a}=\pi_{a} \circ \Psi_{g}^{a}$ and $\Psi_{g} \circ i_{a}=i_{a} \circ \Psi_{g}^{a}$, where $\Psi_{g}: P \rightarrow P$ denotes the action of $g \in G$. Recall also from the standard symplectic reduction theorem that $i_{a}^{*} \Omega=\pi_{a}^{*} \Omega_{a}$ ( $\Omega$ the is symplectic form on $P$ and $\Omega_{a}$ is the symplectic form on $P_{a}$ ). Therefore,

$$
\pi_{a}^{*} \Psi_{g, a}^{*} \Omega_{a}=\left(\Psi_{g}^{a}\right)^{*} \pi_{a}^{*} \Omega_{a}=\left(\Psi_{g}^{a}\right)^{*} i_{a}^{*} \Omega=i_{a}^{*} \Psi_{g}^{*} \Omega=i_{a}^{*} \Omega=\pi_{a}^{*} \Omega_{a} .
$$

Since $\pi_{a}$ is a surjective submersion, we may conclude that

$$
\Psi_{g, a}^{*} \Omega_{a}=\Omega_{a} .
$$

Thus, we have a symplectic action of $G_{a}$ on $P_{a}$.

To show that this resulting action of $G_{a}$ on $P_{a}$ has a momentum map, we first show that the momentum map of the $G$ action restricted to $\mathfrak{g}_{a}$, namely $\mathbf{J}_{S}$ projected to $\mathfrak{g}_{a}^{*}$ induces a well defined map of $P_{a}$ to $\mathfrak{g}_{a}^{*}$. First of all, we restrict $\mathbf{J}_{S}$ to the set $\mathbf{J}_{V}^{-1}(a)$ and project it to $\mathfrak{g}_{a}^{*}$. We claim that this map drops to the quotient space. To check this, note that for $z \in \mathbf{J}_{V}^{-1}(a)$, and $\xi \in \mathfrak{g}_{a}$, equivariance gives us

$$
\left\langle\mathbf{J}_{S}(v z), \xi\right\rangle=\left\langle v \mathbf{J}_{S}(z), \xi\right\rangle=\left\langle(e, v) \mathbf{J}_{S}(z), \xi\right\rangle=\left\langle\mathbf{J}_{S}(z),(e, v)^{-1}(\xi, 0)\right\rangle
$$


Here, the symbol $(e, v)^{-1}(\xi, 0)$ means the adjoint action of the group element $(e, v)^{-1}=(e,-v)$ on the Lie algebra element $(\xi, 0)$. Thus, $(e, v)^{-1}(\xi, 0)=(\xi, \xi v)$, and so, continuing the above calculation, and using the fact that $\mathbf{J}_{V}(z)=a$, we get:

$$
\begin{aligned}
\left\langle\mathbf{J}_{S}(v z), \xi\right\rangle & =\left\langle\mathbf{J}_{S}(z),(e, v)^{-1}(\xi, 0)\right\rangle=\left\langle\mathbf{J}_{S}(z),(\xi, \xi v)\right\rangle \\
& =\left\langle\mathbf{J}_{G}(z), \xi\right\rangle+\left\langle\mathbf{J}_{V}(z), \xi v\right\rangle=\left\langle\mathbf{J}_{G}(z), \xi\right\rangle-\langle\xi a, v\rangle=\left\langle\mathbf{J}_{G}(z), \xi\right\rangle .
\end{aligned}
$$

In this calculation, the term $\langle\xi a, v\rangle$ is zero since $\xi \in \mathfrak{g}_{a}$. Thus, we have shown that the expression

$$
\left\langle\mathbf{J}_{a}([z]), \xi\right\rangle=\left\langle\mathbf{J}_{G}(z), \xi\right\rangle
$$

for $\xi \in \mathfrak{g}_{a}$ is well defined. This expression may be written as

$$
\mathbf{J}_{a} \circ \pi_{a}=\iota_{a}^{*} \circ \mathbf{J}_{G} \circ i_{a}
$$

where $\iota_{a}: \mathfrak{g}_{a} \rightarrow \mathfrak{g}$ is the inclusion map and $\iota_{a}^{*}: \mathfrak{g}^{*} \rightarrow \mathfrak{g}_{a}^{*}$ is its dual.

To show that the map $\mathbf{J}_{a}$ is the momentum map, we first note that for all $\xi \in \mathfrak{g}_{a}$, the vector fields $\xi_{P} \mid\left(\mathbf{J}_{a}^{-1}(a)\right)$ and $\xi_{P_{a}}$ are $\pi_{a}$-related. Thus,

$$
\pi_{a}^{*}\left(\mathbf{i}_{\xi_{P_{a}}} \Omega_{a}\right)=\mathbf{i}_{\xi_{P}} i_{a}^{*} \Omega=i_{a}^{*}\left(\mathbf{i}_{\xi_{P}} \Omega\right)=i_{a}^{*}\left(\mathbf{d}\left\langle\mathbf{J}_{G}, \xi\right\rangle\right)=\pi_{a}^{*}\left(\mathbf{d}\left\langle\mathbf{J}_{a}, \xi\right\rangle\right) .
$$

Again, since $\pi_{a}$ is a surjective submersion, we may conclude that

$$
\mathbf{i}_{\xi_{P a}} \Omega_{a}=\mathbf{d}\left\langle\mathbf{J}_{a}, \xi\right\rangle
$$

and hence $\mathbf{J}_{a}$ is the momentum map for the $G_{a}$ action on $P_{a}$.

Equivariance of $\mathbf{J}_{a}$ follows from that for $\mathbf{J}_{G}$, by a diagram chasing argument as above, using the relation $\mathbf{J}_{a} \circ \pi_{a}=\iota_{a}^{*} \circ \mathbf{J}_{G} \circ i_{a}$ and the relations between the actions of $G$ on $P, \mathbf{J}_{V}^{-1}(a)$ and on $P_{a}$.

Now we turn to the proof of the reduction by stages theorem. Start with the natural inclusion map

$$
j: \mathbf{J}_{S}^{-1}(\sigma) \rightarrow \mathbf{J}_{V}^{-1}(a)
$$

which makes sense since the second component of $\sigma$ is $a$. Composing this map with $\pi_{a}$, the projection of $\mathbf{J}_{V}^{-1}(a)$ to $P_{a}$, we get the map

$$
\pi_{a} \circ j: \mathbf{J}_{S}^{-1}(\sigma) \rightarrow P_{a} .
$$

This map takes values in $\mathbf{J}_{a}^{-1}\left(\mu_{a}\right)$ because of the relation $\mathbf{J}_{a} \circ \pi_{a}=\iota_{a}^{*} \circ \mathbf{J}_{G} \circ i_{a}$ and $\mu_{a}=\iota_{a}^{*}(\mu)$. Thus, we can regard it as a map

$$
\pi_{a} \circ j: \mathbf{J}_{S}^{-1}(\sigma) \rightarrow \mathbf{J}_{a}^{-1}\left(\mu_{a}\right)
$$

Letting $\sigma=(\mu, a)$, there is a group homomorphism $\psi: S_{\sigma} \rightarrow\left(G_{a}\right)_{\mu_{a}}$ defined by projection onto the first factor. The first component $g$ of $(g, v) \in S_{\sigma}$ lies in $\left(G_{a}\right)_{\mu_{a}}$ because

$$
(\mu, a)=(g, v)(\mu, a)=\left(g \mu+\rho_{v}^{*}(g a), g a\right)
$$


implies that, from the second component, that $g \in G_{a}$ and from the first component and the identity $\iota_{a}^{*} \rho_{v}^{*} a=0$ that $g$ also leaves $\mu_{a}$ invariant.

The map $\pi_{a} \circ j$ is equivariant with respect to the action of $S_{\sigma}$ on the domain and the action of $\left(G_{a}\right)_{\mu_{a}}$ on the range via the homomorphism $\psi$. Thus, $\pi_{a} \circ j$ induces a map

$$
\left[\pi_{a} \circ j\right]: P_{\sigma} \rightarrow\left(P_{a}\right)_{\mu_{a}}
$$

Diagram chasing, as above, shows that this map is symplectic.

We will show that this map is a diffeomorphism by finding an inverse. We begin with the construction of a map

$$
\phi: \mathbf{J}_{a}^{-1}\left(\mu_{a}\right) \rightarrow P_{\sigma}
$$

To do this, we first choose an equivalence class $[p]_{a} \in \mathbf{J}_{a}^{-1}\left(\mu_{a}\right) \subset P_{a}$ for $p \in \mathbf{J}_{V}^{-1}(a)$. The equivalence relation is that associated with the map $\pi_{a}$; that is, with the action of $V$. For each such point, we consider a new point $v p$ and will choose $v$ such that $v p \in \mathbf{J}_{S}^{-1}(\sigma)$. For this to hold, we must have

$$
(\mu, a)=\mathbf{J}_{S}(v p)
$$

By equivariance, the right hand side equals

$$
\begin{aligned}
v \mathbf{J}_{S}(p) & =(e, v)\left(\mathbf{J}_{G}(p), \mathbf{J}_{V}(p)\right) \\
& =(e, v)\left(\mathbf{J}_{G}(p), a\right) \\
& =\left(\mathbf{J}_{G}(p)+\rho_{v}^{*}(a), a\right)
\end{aligned}
$$

Thus, we require that

$$
\mu=\mathbf{J}_{G}(p)+\rho_{v}^{*}(a)
$$

This follows from the next lemma.

Lemma 4.5 Denoting the annihilator of $\mathfrak{g}_{a}$ by $\mathfrak{g}_{a}^{o}$, we have

$$
\mathfrak{g}_{a}^{o}=\left\{\rho_{v}^{*} a \mid v \in V\right\}
$$

Proof The identity we showed above, namely $\iota_{a}^{*} \rho_{v}^{*} a=0$, shows that

$$
\mathfrak{g}_{a}^{o} \supset\left\{\rho_{v}^{*} a \mid v \in V\right\}
$$

Now we use the following elementary fact from linear algebra. Let $E$ and $F$ be vector spaces, and $F_{0} \subset F$ be a subspace. Let $T: E \rightarrow F^{*}$ be a linear map whose range lies in the annihilator $F_{0}^{o}$ of $F_{0}$ and that every element $f \in F$ that annihilates the range of $T$ is in $F_{0}$. Then $T$ maps onto $F_{0}^{o}$. (We are phrasing things this way so that the basic framework will also apply in the infinite dimensional case, with the understanding that at this point one would invoke Fredholm type alternative arguments. In the finite dimensional case, the result may be proved by a dimension count.)

In our case, we choose $E=V, F=\mathfrak{g}, F_{0}=\mathfrak{g}_{a}$, and we let $T: V \rightarrow \mathfrak{g}^{*}$ be defined by $T(v)=\rho_{v}^{*}(a)$. To verify the hypothesis of this linear algebra fact, recall that we have already shown that the range of $T$ lies in the annihilator of $\mathfrak{g}_{a}$. Let $\xi \in \mathfrak{g}$ annihilate the range of $T$. Thus, for all $v \in V$,

$$
0=\left\langle\xi, \rho_{v}^{*} a\right\rangle=\left\langle\rho_{v} \xi, a\right\rangle=\langle\xi v, a\rangle=-\langle v, \xi a\rangle
$$


and so $\xi \in \mathfrak{g}_{a}$ as required. Thus, the lemma is proved.

We now apply the lemma to $\mu-\mathbf{J}_{G}(p)$, which lies in the annihilator of $\mathfrak{g}_{a}$ because $\iota_{a}^{*}\left(\mathbf{J}_{G}(p)\right)=\mu_{a}$. Thus, by the lemma, there is a $v$ such that $\mu-\mathbf{J}_{G}(p)=\rho_{v}^{*} a$.

The above argument shows how to construct $v$ so that $v p \in \mathbf{J}_{S}^{-1}(\sigma)$. We continue with the definition of the map $\phi$ by mapping $v p$ to $[v p]_{\sigma}$, its $S_{\sigma}$-equivalence class in $P_{\sigma}$.

To show that the map $\phi$ so constructed is well defined, we replace $p$ by another representative $u p$ of the class $[p]_{a}$; here $u$ is an arbitrary member of $V$. Then choose $v_{1}$ so that $\mathbf{J}_{S}\left(v_{1} u p\right)=\sigma$. Now we must show that $[v p]_{\sigma}=\left[v_{1} u p\right]_{\sigma}$. In other words, we must show that there is a group element $(g, w) \in S_{\sigma}$ such that $(g, w)(e, v) p=\left(e, v_{1}\right)(e, u) p$. This will hold if we can show that $(g, w):=\left(e, v_{1}\right)(e, u)(e, v)^{-1} \in S_{\sigma}$. However, by construction, $\mathbf{J}_{S}(v p)=\sigma=\mathbf{J}_{S}\left(v_{1} u p\right)$; in other words, we have $\sigma=(\mu, a)=(e, v) \mathbf{J}_{S}(p)=\left(e, v_{1}\right)(e, u) \mathbf{J}_{S}(p)$. Thus, by isolating $\mathbf{J}_{S}(p)$, we get $(e, v)^{-1} \sigma=(e, u)^{-1}\left(e, v_{1}\right)^{-1} \sigma$ and so our $(g, w)$ satisfies the required condition. Thus, our map $\phi$ is well defined.

Next we must show that the map $\phi$ is invariant under $\left(G_{a}\right)_{\mu_{a}}$. Thus, let $[p]_{a} \in \mathbf{J}_{a}^{-1}\left(\mu_{a}\right)$ and let $g_{0} \in\left(G_{a}\right)_{\mu_{a}}$. Let $v$ be chosen so that $v p \in \mathbf{J}_{S}^{-1}(\sigma)$ and let $u$ be chosen so that $u g_{0} p \in \mathbf{J}_{S}^{-1}(\sigma)$. We must show that $[v p]_{\sigma}=\left[u g_{0} p\right]_{\sigma}$. In other words, we must find a $(g, w) \in S_{\sigma}$ such that $(g, w)(e, v) p=(e, u)\left(g_{0}, 0\right) p$. This will hold if we can show that $(g, w):=(e, u)\left(g_{0}, 0\right)(e, v)^{-1} \in S_{\sigma}$. But we know that $\sigma=\mathbf{J}_{S}(v p)=\mathbf{J}_{S}\left(u g_{0} p\right)$ or, in other words, by equivariance, $\sigma=(e, v) \mathbf{J}_{S}(p)=$ $(e, u)\left(g_{0}, 0\right) \mathbf{J}_{S}(p)$. By isolating $\mathbf{J}_{S}(p)$, this implies that $(e, v)^{-1} \sigma=\left(g_{0}, 0\right)^{-1}(e, u)^{-1} \sigma$ which means that our $(g, w)$ is indeed in $S_{\sigma}$. Hence $\phi$ is invariant, and so gives a well defined map

$$
[\phi]:\left(P_{a}\right)_{\mu_{a}} \rightarrow P_{\sigma}
$$

Chasing the definitions shows that $[\phi]$ is the inverse of the map $\left[\pi_{\nu} \circ j\right]$. Thus, both maps are symplectic diffeomorphisms. Thus, the reduction by stages theorem is proved.

\section{Acknowledgments}

We thank George Patrick for some helpful comments and for sharing the insightful example in §3.4. We also thank the referees for a careful reading of the manuscript and for their helpful comments.

\section{References}

Aref, H. and Scott W. Jones [1993] Chaotic motion of a solid through ideal fluid Phys. of Fluids A. Fluid Dynamics 5, 3026-3028.

Arnold, V.I. [1993], Dynamical Systems III, Encyclopedia of Mathematics 3, Springer-Verlag, 2nd edition.

Bloch, A.M., P.S. Krishnaprasad, J.E. Marsden, and T.S. Ratiu [1994] Dissipation Induced Instabilities, Ann. Inst. H. Poincaré, Analyse Nonlineare 11, 37-90.

Bloch, A.M., P.S. Krishnaprasad, J.E. Marsden, and T.S. Ratiu [1996] The Euler-Poincaré equations and double bracket dissipation. Comm. Math. Phys. 175, 1-42. 
Bloch, A.M., P.S. Krishnaprasad, J.E. Marsden, and G. Sánchez de Alvarez [1992] Stabilization of rigid body dynamics by internal and external torques, Automatica 28, 745-756.

Bloch, A.M. and J.E. Marsden [1989] Controlling homoclinic orbits, Theoretical and Computational Fluid Mechanics 1, 179-190.

A.M. Bloch, J.E. Marsden and G. Sánchez, Stabilization of relative equilibria of mechanical systems with symmetry, Current and Future Directions in Applied Mathematics, Edited by M. Alber, B. Hu, and J. Rosenthal, Birkhäuser, 1997, 43-64; see also A.M. Bloch, J.E. Marsden, and N. Leonard [1997] Stabilization of Mechanical Systems Using Controlled Lagrangians, preprint.

Coller, B.D. [1995] Suppression of Heteroclinic Bursts in Boundary Layer Models, Ph.D. thesis, Cornell University.

Dellnitz, M., I. Melbourne, and J.E. Marsden [1992] Generic bifurcation of Hamiltonian vector fields with symmetry, Nonlinearity 5, 979-996.

Golubitsky, M., J.E. Marsden, I. Stewart, and M. Dellnitz [1995] The constrained Liapunov Schmidt procedure and periodic orbits. Fields Institute Comm. 4, 81-127.

Haller, G. [1992] Gyroscopic stability and its loss in systems with two essential coordinates, Int. J. Nonlinear Mech. 27, 113-127.

Holm, D.D., J.E. Marsden, T.S. Ratiu, and A. Weinstein [1985] Nonlinear stability of fluid and plasma equilibria, Phys. Rep. 123, 1-116.

Knobloch, E., A. Mahalov, and J.E. Marsden [1994] Normal Forms for three-dimensional Parametric Instabilities in Ideal Hydrodynamics Physica D 73, 49-81.

Krishnaprasad, P.S. [1989] Eulerian many-body problems, Cont. Math. AMS 97, 187-208.

Lamb, H. [1932] Hydrodynamics, Dover, 6th edition.

Landsman, N.P. [1995] Rieffel induction as generalized quantum Marsden-Weinstein reduction. J. Geom. and Phys. 15, 285-319.

Langford, W.F. and K. Zhan [1994] Strongly resonant Hopf bifurcations and vortex-induced vibrations. Nonlinearity and Chaos in Engineering Dynamics, J.M.T. Thompson and S.R. Bishop, eds., Wiley, 241-248.

Leonard, N.E. [1995a] Control synthesis and adaptation for an underactuated autonomous underwater vehicle, IEEE J. of Oceanic Eng. 20, 211-220.

Leonard, N.E. [1995] Stability of a bottom-heavy underwater vehicle, Automatica 33, March 1997.

Leonard, N.E. [1995c] Periodic forcing, dynamics and control of underactuated spacecraft and underwater vehicles, Proc. 34th IEEE Conference on Decision and Control, 3980-3985.

Leonard, N.E. [1996] Stabilization of steady motions of an underwater vehicle, Proc. 35th IEEE Conference on Decision and Control, 961-966. 
Leonard, N.E. and P.S. Krishnaprasad [1995] Motion control of drift-free, left-invariant systems on Lie groups, IEEE Trans. Automatic Control 40, 1539-1554.

Lewis, D., T.S. Ratiu, J.C. Simo, and J.E. Marsden [1992] The heavy top, a geometric treatment, Nonlinearity 5, 1-48.

Libermann, P. and C.M. Marle [1987] Symplectic Geometry and Analytical Mechanics. Kluwer Academic Publishers.

Marsden, J.E. [1992], Lectures on Mechanics London Mathematical Society Lecture note series, 174, Cambridge University Press.

Marsden, J.E. and T.S. Ratiu [1994] Introduction to Mechanics and Symmetry. Texts in Applied Mathematics, 17, Springer-Verlag.

Marsden, J.E. and T.S. Ratiu [1996] Reduction by stages, in preparation.

Marsden, J.E., T.S. Ratiu, and A. Weinstein [1984] Semidirect products and reduction in mechanics, Trans. Am. Math. Soc. 281, 147-177.

Novikov, S.P. and I. Shmel'tser [1982] Periodic solutions of Kirchhoff's equations for the free motion of a rigid body in a fluid and the extended theory of Lyusternik-Shnirel'man-Morse I, Funct. An. and Appl. 15, 197-207.

Patrick, G. [1992] Relative equilibria in Hamiltonian systems: The dynamic interpretation of nonlinear stability on a reduced phase space, J. Geom. and Phys. 9, 111-119.

Patrick, G. [1995] Relative equilibria of Hamiltonian systems with symmetry: linearization, smoothness and drift, J. Nonlinear Sci. 5, 373-418.

Simo, J.C., D.R. Lewis, and J.E. Marsden [1991] Stability of relative equilibria I: The reduced energy momentum method, Arch. Rat. Mech. Anal. 115, 15-59.

Sjamaar, R. and E. Lerman [1991] Stratified symplectic spaces and reduction, Ann. of Math. 134, $375-422$.

van der Meer, J.C. [1985] The Hamiltonian Hopf Bifurcation. Springer Lecture Notes in Mathematics $\mathbf{1 1 6 0 .}$

van der Meer, J.C. [1990] Hamiltonian Hopf bifurcation with symmetry, Nonlinearity 3, 10411056.

Weinstein, A. [1984] Stability of Poisson-Hamilton equilibria, Cont. Math. AMS 28, 3-14. 